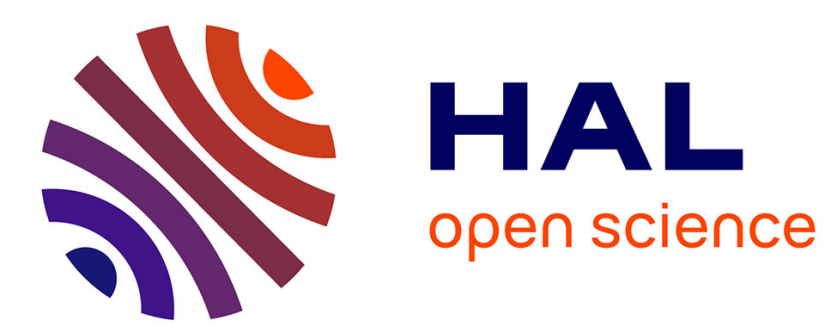

\title{
Global representation and multi-scale expansion for the Dirichlet problem in a domain with a small hole close to the boundary
}

Virginie Bonnaillie-Noël, Matteo Dalla, Marc Dambrine, Paolo Musolino

\section{- To cite this version:}

Virginie Bonnaillie-Noël, Matteo Dalla, Marc Dambrine, Paolo Musolino. Global representation and multi-scale expansion for the Dirichlet problem in a domain with a small hole close to the boundary. 2019. hal-02176154

\author{
HAL Id: hal-02176154 \\ https://hal.science/hal-02176154
}

Preprint submitted on 7 Jul 2019

HAL is a multi-disciplinary open access archive for the deposit and dissemination of scientific research documents, whether they are published or not. The documents may come from teaching and research institutions in France or abroad, or from public or private research centers.
L'archive ouverte pluridisciplinaire HAL, est destinée au dépôt et à la diffusion de documents scientifiques de niveau recherche, publiés ou non, émanant des établissements d'enseignement et de recherche français ou étrangers, des laboratoires publics ou privés. 


\title{
Global representation and multi-scale expansion for the Dirichlet problem in a domain with a small hole close to the boundary
}

\author{
Virginie Bonnaillie-Noël, Matteo Dalla Riva! \\ Marc Dambrine; and Paolo Musolino ${ }^{\ddagger}$
}

February 22, 2019

\begin{abstract}
For each pair $\varepsilon=\left(\varepsilon_{1}, \varepsilon_{2}\right)$ of positive parameters, we define a perforated domain $\Omega_{\varepsilon}$ by making a small hole of size $\varepsilon_{1} \varepsilon_{2}$ in an open regular subset $\Omega$ of $\mathbb{R}^{n}(n \geq 3)$. The hole is situated at distance $\varepsilon_{1}$ from the outer boundary $\partial \Omega$ of the domain. Then, when $\varepsilon \rightarrow(0,0)$ both the size of the hole and its distance from $\partial \Omega$ tend to zero, but the size shrinks faster than the distance. In such perforated domain $\Omega_{\varepsilon}$ we consider a Dirichlet problem for the Laplace equation and we denote by $u_{\varepsilon}$ its solution. Our aim is to represent the map that takes $\varepsilon$ to $u_{\varepsilon}$ in term of real analytic functions of $\varepsilon$ defined in a neighborhood of $(0,0)$. In contrast with previous results valid only for restrictions of $u_{\varepsilon}$ to suitable subsets of $\Omega_{\varepsilon}$, we prove a global representation formula that holds on the whole of $\Omega_{\varepsilon}$. Such a formula allows to rigorously justify multi-scale expansions, which we subsequently construct.
\end{abstract}

Keywords: Dirichlet problem; singularly perturbed perforated domain; Laplace operator; real analytic continuation in Banach space; multi-scale asymptotic expansion

2010 Mathematics Subject Classification: 35J25; 31B10; 45A05; 35B25; 35C20

\section{Introduction}

Boundary value problems in domains with small holes are a typical subject of asymptotic analysis and are usually studied by means of asymptotic expansion methods. The aim of such methods is to obtain an asymptotic approximation that describes the behavior of the solution as a small perturbation parameter (which could be the size of the hole) tends to zero. There are different techniques to obtain such approximations: for example, the method of matching

\footnotetext{
* Département de mathématiques et applications, École normale supérieure, CNRS, PSL University, 45 rue d'Ulm, 75005 Paris, France bonnaillie@math.cnrs.fr

${ }^{\dagger}$ Department of Mathematics, The University of Tulsa, 800 South Tucker Drive, Tulsa, Oklahoma 74104, USA matteo.dallariva@gmail.com

${ }^{\ddagger}$ Laboratoire de Matheḿatiques et de leurs Applications, UMR 5142, CNRS, Université de Pau et des Pays de l'Adour, av. de l'Université, BP 1155, F-64013 Pau Cedex, France Marc.Dambrine@univ-pau.fr

${ }^{\S}$ Dipartimento di Matematica 'Tullio Levi-Civita', Università degli Studi di Padova, via Trieste 63, 35121 Padova, Italy musolino@math.unipd.it
} 
outer and inner expansions of Il'in [9, 10, the multi-scale (or compound) expansion method of Maz'ya, Nazarov, and Plamenevskij [14], and the asymptotic analysis of Green's kernels of Maz'ya, Movchan, and Nieves [13].

Recently another method has appeared that takes a different stand point with respect to asymptotic analysis. This new method is based on the functional analytic approach proposed by Lanza de Cristoforis (cf. [11, 12]) and the core feature is the description of the solution in terms of real analytic functions of one or several variables depending on the small parameter that characterizes the perturbation.

In this paper we show an interaction between the functional analytic approach and the expansion methods of asymptotic analysis. To do so, we will consider a Dirichlet problem for the Laplace equation in a domain with a small hole 'moderately close' to the boundary. That is, a hole that approaches the outer boundary of the domain while shrinking its size at a faster speed.

The same problem has been already considered in [1], where the functional analytic approach has been used to prove real analytic representation formulas for certain restrictions of the solution to suitable subsets of the perturbed domain. Here instead, we obtain a representation formula in the whole domain of definition of the solution. The advantage of such a formula is that it can be used to justify multi-scale expansions and to deduce an effective recursive algorithm for the computation of such expansions.

We now introduce the geometric setting of the problem. For the sake of simplicity, we confine ourselves to the case where the dimension $n \in \mathbb{N}$ is greater than or equal to 3 (in contrast with [1], where the two dimensional case is also treated). Moreover, without loss of generality, we place the problem in the upper half-space

$$
\mathbb{R}_{+}^{n} \equiv\left\{\mathrm{x}=\left(x_{1}, \ldots, x_{n}\right) \in \mathbb{R}^{n}: x_{n}>0\right\} .
$$

We note that the boundary $\partial \mathbb{R}_{+}^{n}$ coincides with the hyperplane $x_{n}=0$. Then we fix a domain $\Omega$ that plays the role of the 'unperturbed' domain in which we make a hole. We assume that

$$
\Omega \text { is an open bounded connected subset of } \mathbb{R}_{+}^{n} \text { of class } \mathscr{C}^{1, \alpha},
$$

where $\alpha \in] 0,1[$ is a regularity parameter. The definition of functions and sets of the usual Schauder classes $\mathscr{C}^{k, \alpha}(k=0,1)$ can be found, for example, in Gilbarg and Trudinger [8, §6.2]. In this paper, we assume that a part of the boundary $\partial \Omega$ of $\Omega$ is flat and that the hole is approaching it (see Figure 1). To do so, we set

$$
\partial_{0} \Omega \equiv \partial \Omega \cap \partial \mathbb{R}_{+}^{n}, \quad \partial_{+} \Omega \equiv \partial \Omega \cap \mathbb{R}_{+}^{n},
$$

and we assume that

$$
\partial_{0} \Omega \text { is an open neighborhood of } 0 \text { in } \partial \mathbb{R}_{+}^{n} .
$$

Then we introduce another set $\omega$ such that

$$
\omega \text { is a bounded open connected subset of } \mathbb{R}^{n} \text { of class } \mathscr{C}^{1, \alpha} \text { and } 0 \in \omega \text {. }
$$

$\omega$ plays the role of a reference set for the shape of the perforation. Finally, we fix a point

$$
\mathrm{p}=\left(p_{1}, \ldots, p_{n}\right) \in \mathbb{R}_{+}^{n},
$$


and define the hole $\omega_{\varepsilon}$ by

$$
\omega_{\varepsilon} \equiv \varepsilon_{1} \mathrm{p}+\varepsilon_{1} \varepsilon_{2} \omega, \quad \forall \varepsilon \equiv\left(\varepsilon_{1}, \varepsilon_{2}\right) \in \mathbb{R}^{2} .
$$

We adopt the following notation. If $\varepsilon^{\prime} \equiv\left(\varepsilon_{1}^{\prime}, \varepsilon_{2}^{\prime}\right), \varepsilon^{\prime \prime} \equiv\left(\varepsilon_{1}^{\prime \prime}, \varepsilon_{2}^{\prime \prime}\right) \in \mathbb{R}^{2}$, then we write $\varepsilon^{\prime} \leq \varepsilon^{\prime \prime}$ (respectively, $\varepsilon^{\prime}<\varepsilon^{\prime \prime}$ ) if and only if $\varepsilon_{j}^{\prime} \leq \varepsilon_{j}^{\prime \prime}$ (respectively, $\varepsilon_{j}^{\prime}<\varepsilon_{j}^{\prime \prime}$ ), for $j=1,2$, and denote by ]$\varepsilon^{\prime}, \varepsilon^{\prime \prime}\left[\right.$ the open rectangular domain of $\varepsilon \in \mathbb{R}^{2}$ such that $\varepsilon^{\prime}<\varepsilon<\varepsilon^{\prime \prime}$. We also set $\mathbf{0} \equiv(0,0)$. Then there exists $\varepsilon^{\text {ad }}>\mathbf{0}$ such that

$$
\left.\overline{\omega_{\varepsilon}} \subseteq \Omega, \quad \forall \varepsilon \in\right] \mathbf{0}, \varepsilon^{\mathrm{ad}}[.
$$

The rectangular set $] \mathbf{0}, \varepsilon^{\text {ad }}$ [ consists of admissible parameters for which we can define the perforated domain $\Omega_{\varepsilon}$ obtained by removing from the unperturbed domain $\Omega$ the closure $\overline{\omega_{\varepsilon}}$ of $\omega_{\varepsilon}$, i.e.,

$$
\left.\Omega_{\varepsilon} \equiv \Omega \backslash \overline{\omega_{\varepsilon}}, \quad \forall \varepsilon \in\right] \mathbf{0}, \varepsilon^{\mathrm{ad}}[.
$$

It can be easily verified that for all $\varepsilon \in] \mathbf{0}, \varepsilon^{\text {ad }}\left[, \Omega_{\varepsilon}\right.$ is a bounded connected open domain of class $\mathscr{C}^{1, \alpha}$ with boundary $\partial \Omega_{\varepsilon}$ consisting of two connected components: $\partial \Omega$ and $\partial \omega_{\varepsilon}=\varepsilon_{1} p+\varepsilon_{1} \varepsilon_{2} \partial \omega$. The parameter $\varepsilon_{1}$ controls the distance of the hole $\omega_{\varepsilon}$ from the boundary $\partial \Omega$; the product $\varepsilon_{1} \varepsilon_{2}$ controls its size. As the pair $\left.\varepsilon \in\right] \mathbf{0}, \varepsilon^{\text {ad }}$ [ approaches the singular value $\mathbf{0}$, both the size of the cavity and its distance from the boundary $\partial \Omega$ tend to 0 . In particular, the ratio of the size of the hole to its distance from the boundary tends to 0 , and we can say that the size tends to zero 'faster' than the distance. Figure 1 illustrates our geometric setting.

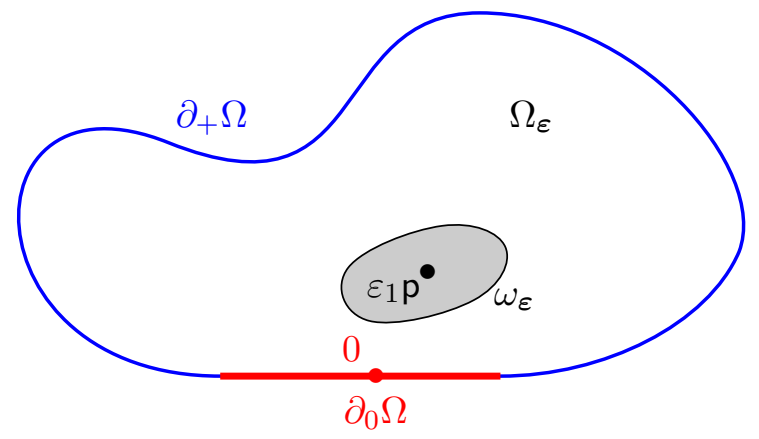

Figure 1: Geometric setting.

Now, we fix two functions $g^{\mathrm{o}} \in \mathscr{C}^{1, \alpha}(\partial \Omega)$ and $g^{\mathrm{i}} \in \mathscr{C}^{1, \alpha}(\partial \omega)$ and for each $\left.\varepsilon \in\right] \mathbf{0}, \boldsymbol{\varepsilon}^{\text {ad }}$ [ we consider the following Dirichlet problem in the $\varepsilon$-dependent domain $\Omega_{\varepsilon}$ :

$$
\begin{cases}\Delta u(\mathrm{x})=0, & \forall \mathrm{x} \in \Omega_{\varepsilon}, \\ u(\mathrm{x})=g^{\mathrm{o}}(\mathrm{x}), & \forall \mathrm{x} \in \partial \Omega, \\ u(\mathrm{x})=g^{\mathrm{i}}\left(\frac{\mathrm{x}-\varepsilon_{1} \mathrm{p}}{\varepsilon_{1} \varepsilon_{2}}\right), & \forall \mathrm{x} \in \partial \omega_{\varepsilon} .\end{cases}
$$

As is well known, (1.1) has a unique solution in $\mathscr{C}^{1, \alpha}\left(\overline{\Omega_{\varepsilon}}\right)$ (which depends on $\varepsilon$ ) and we denote such a solution by $u_{\varepsilon}$. Our aim is to understand the behavior of $u_{\varepsilon}$ when the parameter $\boldsymbol{\varepsilon}=\left(\varepsilon_{1}, \varepsilon_{2}\right)$ approaches the singular value $\mathbf{0} \equiv(0,0)$ and study its dependence upon $\boldsymbol{\varepsilon}$. As mentioned above, we proceed in two steps: 
- First we use the functional analytic approach to describe the dependence of $u_{\varepsilon}$ upon $\varepsilon$ in terms of real analytic maps of $\varepsilon$ defined in a neighborhood of $\mathbf{0}$. In particular, we obtain a global representation formula that holds in the whole of $\overline{\Omega_{\varepsilon}}$.

- Then we exploit such a formula to deduce a rigorously justified multi-scale iterative procedure and compute an asymptotic expansion of $u_{\varepsilon}$ for $\varepsilon$ close to $\mathbf{0}$.

\subsection{The Functional Analytic Approach}

As we have seen in [1], to apply the functional analytic approach to problem (1.1), it is necessary to introduce further regularity conditions on the domain $\Omega$ (in addition to $\left(H_{1}\right)$ and $\left(\mathrm{H}_{2}\right.$ ) and on the Dirichlet datum $g^{\mathrm{o}}$. Namely, we have to ask that

$$
\overline{\partial_{+} \Omega} \text { is a compact submanifold with boundary of } \mathbb{R}^{n} \text { of class } \mathscr{C}^{1, \alpha} \text {, }
$$

and that

$$
\text { there exists } r_{0}>0 \text { such that the restriction } g_{\mid \mathcal{B}\left(0, r_{0}\right) \cap \partial_{0} \Omega}^{\mathrm{o}} \text { is real analytic. }
$$

Here and in what follows $\mathcal{B}\left(0, r_{0}\right)$ denotes the ball in $\mathbb{R}^{n}$ of radius $r_{0}$ and center 0 . A consequence of $\left(\mathrm{H}_{3}\right)$ is that there exist linear and continuous extension operators $E^{k, \alpha}$ from $\mathscr{C}^{k, \alpha}\left(\overline{\partial_{+} \Omega}\right)$ to $\mathscr{C}^{k, \alpha}(\partial \Omega)$, for $k=0,1$ (cf. [1, Lemma 2.17]).

Under assumptions $\left(H_{1}\right)-\left(H_{4}\right)$, we have proven that the restriction of $u_{\varepsilon}$ to a subset 'far' from the hole depends real analytically on the perturbation parameters $\varepsilon$, as the following theorem states (see [1, Thm. 1.2]).

Theorem 1.1. Let $\Omega^{\prime}$ be an open subset of $\Omega$ such that $0 \notin \overline{\Omega^{\prime}}$. There are $\left.\varepsilon^{\prime} \in\right] \mathbf{0}, \varepsilon^{\text {ad }}[$ with $\overline{\omega_{\varepsilon}} \cap \overline{\Omega^{\prime}}=\emptyset$ for all $\left.\varepsilon \in\right]-\varepsilon^{\prime}, \varepsilon^{\prime}\left[\right.$ and a real analytic map $\mathfrak{U}_{\Omega^{\prime}}$ from $]-\boldsymbol{\varepsilon}^{\prime}, \boldsymbol{\varepsilon}^{\prime}\left[\right.$ to $\mathscr{C}^{1, \alpha}\left(\overline{\Omega^{\prime}}\right)$ such that

$$
\left.u_{\varepsilon \mid \overline{\Omega^{\prime}}}=\mathfrak{U}_{\Omega^{\prime}}[\varepsilon], \quad \forall \varepsilon \in\right] \mathbf{0}, \boldsymbol{\varepsilon}^{\prime}[
$$

Furthermore,

$$
\mathfrak{U}_{\Omega^{\prime}}[\mathbf{0}]=u_{\mathbf{0} \mid \overline{\Omega^{\prime}}},
$$

where $u_{\mathbf{0}} \in \mathscr{C}^{1, \alpha}(\bar{\Omega})$ is the unique solution of

$$
\begin{cases}\Delta u_{0}=0 & \text { in } \Omega, \\ u_{\mathbf{0}}=g^{\mathrm{o}} & \text { on } \partial \Omega .\end{cases}
$$

The theorem above characterizes the 'macroscopic' behavior of the solution 'far' from the hole. A similar result has been proven also for the 'microscopic' behavior of $u_{\varepsilon}$ close to the perforation, i.e. for the behavior of the rescaled function $u_{\varepsilon}\left(\varepsilon_{1} \mathrm{p}+\varepsilon_{1} \varepsilon_{2} \cdot\right)$ (see [1, Thm. 3.6]).

Theorem 1.2. Let $\omega^{\prime}$ be an open bounded subset of $\mathbb{R}^{n} \backslash \bar{\omega}$. There are $\left.\varepsilon^{\prime \prime} \in\right] \mathbf{0}, \varepsilon^{\text {ad }}[$ with $\left(\varepsilon_{1} \mathrm{p}+\varepsilon_{1} \varepsilon_{2} \overline{\omega^{\prime}}\right) \subseteq \mathcal{B}\left(0, r_{1}\right)$ for all $\left.\varepsilon \in\right]-\varepsilon^{\prime \prime}, \varepsilon^{\prime \prime}\left[\right.$ and a real analytic map $\mathfrak{V}_{\omega^{\prime}}$ from $]-\varepsilon^{\prime \prime}, \varepsilon^{\prime \prime}[$ to $\mathscr{C}^{1, \alpha}\left(\overline{\omega^{\prime}}\right)$ such that

$$
\left.u_{\varepsilon}\left(\varepsilon_{1} \mathrm{p}+\varepsilon_{1} \varepsilon_{2} \cdot\right)_{\mid \overline{\omega^{\prime}}}=\mathfrak{V}_{\omega^{\prime}}[\varepsilon], \quad \forall \varepsilon \in\right] \mathbf{0}, \varepsilon^{\prime \prime}[.
$$

Furthermore,

$$
\mathfrak{V}_{\omega^{\prime}}[\mathbf{0}]=v_{\mathbf{0} \mid \overline{\omega^{\prime}}}
$$


where $v_{\mathbf{0}} \in \mathscr{C}_{\mathrm{loc}}^{1, \alpha}\left(\mathbb{R}^{n} \backslash \omega\right)$ is the unique solution of

$$
\begin{cases}\Delta v_{\mathbf{0}}=0 & \text { in } \mathbb{R}^{n} \backslash \omega \\ v_{\mathbf{0}}=g^{\mathrm{i}} & \text { on } \partial \omega \\ \lim _{\mathrm{X} \rightarrow \infty} v_{\mathbf{0}}(\mathrm{X})=g^{\mathrm{o}}(0) . & \end{cases}
$$

\subsection{Main results}

Theorems 1.1 and 1.2 deal with the 'local behavior' of the solution, either 'far' from or 'close' to the hole. The first aim of this paper is to prove a global representation formula that holds on the whole of $\overline{\Omega_{\varepsilon}}$. In particular, up to the boundary of the hole and up to the outer boundary.

Such a representation formula is presented in Theorem 1.3 here below. To state it correctly, we first need to introduce suitable Banach spaces of harmonic functions in $\Omega$ and in the exterior domain $\mathbb{R}^{n} \backslash \bar{\omega}$. Then we set

$$
\begin{aligned}
\mathscr{C}_{\mathrm{h}}^{1, \alpha}(\bar{\Omega}) & \equiv\left\{v \in \mathscr{C}^{1, \alpha}(\bar{\Omega}): \Delta v=0 \text { in } \Omega\right\}, \\
\mathscr{C}_{\mathrm{h}}^{1, \alpha}\left(\mathbb{R}^{n} \backslash \omega\right) & \equiv\left\{v \in \mathscr{C}_{\mathrm{loc}}^{1, \alpha}\left(\mathbb{R}^{n} \backslash \omega\right): \Delta v=0 \text { in } \mathbb{R}^{n} \backslash \bar{\omega}, \text { and } \lim _{\mathrm{x} \rightarrow \infty} v(\mathrm{x})=0\right\} .
\end{aligned}
$$

We observe that $\mathscr{C}_{\mathrm{h}}^{1, \alpha}(\bar{\Omega})$ is a closed subspace of $\mathscr{C}^{1, \alpha}(\bar{\Omega})$, and accordingly it is a Banach space with the norm induced by $\mathscr{C}^{1, \alpha}(\bar{\Omega})$. A similar argument does not work for $\mathscr{C}_{\mathrm{h}}^{1, \alpha}\left(\mathbb{R}^{n} \backslash \omega\right)$, because $\mathscr{C}_{\text {loc }}^{1, \alpha}\left(\mathbb{R}^{n} \backslash \omega\right)$ is not a normed space, but a Fréchet space (cf. Definition 2.2 below). Then we recall that the solution of a Dirichlet problem in $\mathbb{R}^{n} \backslash \omega$ which decays at infinity exists and is unique (cf., e.g., Folland [7, Chap. 2], note that here $n \geq 3$ ). Accordingly, the map which takes a function $v$ to its trace $v_{\mid \partial \omega}$ is a linear isomorphism between $\mathscr{C}_{\mathrm{h}}^{1, \alpha}\left(\mathbb{R}^{n} \backslash \omega\right)$ and $\mathscr{C}^{1, \alpha}(\partial \omega)$ and we can define a norm on $\mathscr{C}_{\mathrm{h}}^{1, \alpha}\left(\mathbb{R}^{n} \backslash \omega\right)$ by setting

$$
\|v\|_{\mathscr{C}_{\mathrm{h}}^{1, \alpha}\left(\mathbb{R}^{n} \backslash \omega\right)} \equiv\left\|v_{\mid \partial \omega}\right\|_{\mathscr{C}^{1, \alpha}(\partial \omega)}, \quad \forall v \in \mathscr{C}_{\mathrm{h}}^{1, \alpha}\left(\mathbb{R}^{n} \backslash \omega\right)
$$

With such a norm $\mathscr{C}_{\mathrm{h}}^{1, \alpha}\left(\mathbb{R}^{n} \backslash \omega\right)$ is a Banach space. In addition, exploiting elliptic a priori estimates, one can verify that the topology generated by $\|\cdot\|_{\mathscr{C}_{\mathrm{h}}^{1, \alpha}\left(\mathbb{R}^{n} \backslash \omega\right)}$ is equivalent to the one induced by the embedding in (the Fréchet space) $\mathscr{C}_{\text {loc }}^{1, \alpha}\left(\mathbb{R}^{n} \backslash \omega\right)$.

We can now state our global real analyticity representation theorem.

Theorem 1.3. There exist $\left.\varepsilon^{*} \in\right] \mathbf{0}, \varepsilon^{\text {ad }}[$ and two univocally identified real analytic maps $\mathfrak{U}$ from $]-\varepsilon^{*}, \varepsilon^{*}\left[\right.$ to $\mathscr{C}_{\mathrm{h}}^{1, \alpha}(\bar{\Omega})$ and $\mathfrak{V}$ from $]-\varepsilon^{*}, \varepsilon^{*}\left[\right.$ to $\mathscr{C}_{\mathrm{h}}^{1, \alpha}\left(\mathbb{R}^{n} \backslash \omega\right)$ such that

$$
\left.u_{\varepsilon}(\mathrm{x})=\mathfrak{U}[\varepsilon](\mathrm{x})+\mathfrak{V}[\varepsilon]\left(\frac{\mathrm{x}-\varepsilon_{1} \mathrm{p}}{\varepsilon_{1} \varepsilon_{2}}\right)-\mathfrak{V}[\varepsilon]\left(\frac{\varsigma(\mathrm{x})-\varepsilon_{1} \mathrm{p}}{\varepsilon_{1} \varepsilon_{2}}\right), \quad \forall \mathrm{x} \in \overline{\Omega_{\varepsilon}}, \varepsilon \in\right] \mathbf{0}, \varepsilon^{*}[,
$$

where $\varsigma$ denotes the reflexion with respect to the hyperplane $\partial \mathbb{R}_{+}^{n}$. That is,

$$
\varsigma(\mathrm{x}) \equiv\left(x_{1}, \ldots, x_{n-1},-x_{n}\right), \quad \forall \mathrm{x}=\left(x_{1}, \ldots, x_{n}\right) \in \mathbb{R}^{n} .
$$

In addition, we have

$$
\mathfrak{U}[\mathbf{0}]=u_{\mathbf{0}} \quad \text { and } \quad \mathfrak{V}[\mathbf{0}]=v_{\mathbf{0}}-g^{\mathrm{o}}(0),
$$

with $u_{\mathbf{0}} \in \mathscr{C}_{\mathrm{h}}^{1, \alpha}(\bar{\Omega})$ and $v_{\mathbf{0}} \in \mathscr{C}_{\mathrm{h}}^{1, \alpha}\left(\mathbb{R}^{n} \backslash \omega\right)$ solutions of 1.2 and $(1.4)$, respectively. 
A consequence of Theorem 1.3 is that there exist $\left.\varepsilon^{* *} \in\right] \mathbf{0}, \varepsilon^{*}[$ and two countable families of functions $\left\{U_{i, j}\right\}_{i, j \in \mathbb{N}^{2}} \subseteq \mathscr{C}_{\mathrm{h}}^{1, \alpha}(\bar{\Omega})$ and $\left\{V_{i, j}\right\}_{i, j \in \mathbb{N}^{2}} \subseteq \mathscr{C}_{\mathrm{h}}^{1, \alpha}\left(\mathbb{R}^{n} \backslash \omega\right)$ such that the series

$$
\sum_{i, j \in \mathbb{N}^{2}} \varepsilon_{1}^{i} \varepsilon_{2}^{j} U_{i, j} \quad \text { and } \quad \sum_{i, j \in \mathbb{N}^{2}} \varepsilon_{1}^{i} \varepsilon_{2}^{j} V_{i, j}
$$

converge for $\varepsilon \in]-\varepsilon^{* *}, \varepsilon^{* *}\left[\right.$ in $\mathscr{C}_{\mathrm{h}}^{1, \alpha}(\bar{\Omega})$ and in $\mathscr{C}_{\mathrm{h}}^{1, \alpha}\left(\mathbb{R}^{n} \backslash \omega\right)$, respectively, and such that

$$
u_{\varepsilon}(\mathrm{x})=\sum_{i, j \in \mathbb{N}^{2}} \varepsilon_{1}^{i} \varepsilon_{2}^{j} U_{i, j}(\mathrm{x})+\sum_{i, j \in \mathbb{N}^{2}} \varepsilon_{1}^{i} \varepsilon_{2}^{j} V_{i, j}\left(\frac{\mathrm{x}-\varepsilon_{1} \mathrm{p}}{\varepsilon_{1} \varepsilon_{2}}\right)-\sum_{i, j \in \mathbb{N}^{2}} \varepsilon_{1}^{i} \varepsilon_{2}^{j} V_{i, j}\left(\frac{\varsigma(\mathrm{x})-\varepsilon_{1} \mathrm{p}}{\varepsilon_{1} \varepsilon_{2}}\right),
$$

for all $\mathrm{x} \in \overline{\Omega_{\varepsilon}}$ and all $\left.\varepsilon \in\right] \mathbf{0}, \varepsilon^{* *}[$. Moreover, by the uniqueness of $\mathfrak{U}$ and $\mathfrak{V}$ and by the identity principle for real analytic functions, one verifies that such families $\left\{U_{i, j}\right\}_{i, j \in \mathbb{N}^{2}}$ and $\left\{V_{i, j}\right\}_{i, j \in \mathbb{N}^{2}}$ are univocally identified by (1.7).

Another consequence of the representation $(1.5)$ is that we can approximate the solution $u_{\varepsilon}$ by means of a sum of slow variable terms (with coefficients evaluated at $\mathrm{x}$ ), fast variable terms (with coefficients evaluated at $\left(x-\varepsilon_{1} p\right) /\left(\varepsilon_{1} \varepsilon_{2}\right)$ ), and of what we can call 'reflected fast variable terms' (with coefficients evaluated at $\left(\varsigma(\mathrm{x})-\varepsilon_{1} \mathrm{p}\right) /\left(\varepsilon_{1} \varepsilon_{2}\right)$ ). In particular we have the following.

Corollary 1.4. Let $\left\{U_{i, j}\right\}_{i, j \in \mathbb{N}^{2}} \subseteq \mathscr{C}_{\mathrm{h}}^{1, \alpha}(\bar{\Omega})$ and $\left\{V_{i, j}\right\}_{i, j \in \mathbb{N}^{2}} \subseteq \mathscr{C}_{\mathrm{h}}^{1, \alpha}\left(\mathbb{R}^{n} \backslash \omega\right)$ be as in (1.7). Let $u_{\mathbf{0}} \in \mathscr{C}_{\mathrm{h}}^{1, \alpha}(\bar{\Omega})$ and $v_{\mathbf{0}} \in \mathscr{C}_{\mathrm{h}}^{1, \alpha}\left(\mathbb{R}^{n} \backslash \omega\right)$ be the solutions of $(1.2)$ and $(1.4)$, respectively. Then, for $\varepsilon \rightarrow \mathbf{0}$ the asymptotic approximation

$$
\begin{aligned}
u_{\boldsymbol{\varepsilon}}(\mathrm{x})= & u_{\mathbf{0}}(\mathrm{x})+v_{\mathbf{0}}\left(\frac{\mathrm{x}-\varepsilon_{1} \mathrm{p}}{\varepsilon_{1} \varepsilon_{2}}\right)-v_{\mathbf{0}}\left(\frac{\varsigma(\mathrm{x})-\varepsilon_{1} \mathrm{p}}{\varepsilon_{1} \varepsilon_{2}}\right)+\sum_{0<i+j \leq N} \varepsilon_{1}^{i} \varepsilon_{2}^{j} U_{i, j}(\mathrm{x}) \\
& +\sum_{0<i+j \leq N} \varepsilon_{1}^{i} \varepsilon_{2}^{j} V_{i, j}\left(\frac{\mathrm{x}-\varepsilon_{1} \mathrm{p}}{\varepsilon_{1} \varepsilon_{2}}\right)-\sum_{0<i+j \leq N} \varepsilon_{1}^{i} \varepsilon_{2}^{j} V_{i, j}\left(\frac{\varsigma(\mathrm{x})-\varepsilon_{1} \mathrm{p}}{\varepsilon_{1} \varepsilon_{2}}\right)+O\left(\sum_{i+j=N+1} \varepsilon_{1}^{i} \varepsilon_{2}^{j}\right)
\end{aligned}
$$

holds uniformly in $\mathrm{x} \in \overline{\Omega_{\varepsilon}}$.

As we shall see, the coefficients $U_{i, j}$ and $V_{i, j}$ are also univocally identified by the approximation (1.8). Specifically, any approximation in the form of (1.8) where the coefficients $U_{i, j}$ and $V_{i, j}$ are continuous functions and the $V_{i, j}$ 's vanish for $\mathrm{x} \rightarrow \infty$, must coincide with the one that we derive from Theorem 1.3 (cf. Proposition 3.2 below). Moreover, with the exception of certain particular cases, one can see that there are no asymptotic approximations consisting solely of slow variable and fast variable terms but no reflected fast variable terms. At least, no reasonable approximations with a remainder in $o\left(\varepsilon_{1}+\varepsilon_{2}\right)$ or smaller (cf. Example 3.3 below and the remarks right after it).

The presence of such reflected fast variable terms is ultimately due to the two parameters perturbation considered in the paper, which uncouples the size of the hole from its distance from the boundary. They would not have appeared if one of the parameters, say $\varepsilon_{2}$, was 'frozen' and the perturbations for different values of $\varepsilon_{1}$ were locally similar one to another.

We also mention that a global analytic representation was obtained in the paper [3] with Costabel and Dauge. Such paper deals with a Dirichlet problem for the Poisson equation in a 
plane sector domain and analyzes the effect of a self-similar perturbation close to the vertex. In such a case, no reflected fast variable terms showed up.

Having proved the existence of asymptotic approximations of $u_{\varepsilon}$ in the form (1.4), our second aim in the paper is to deduce a strategy based on the multi-scale expansion method to compute the coefficients $U_{i, j}$ and $V_{i, j}$ up to the solution of certain auxiliary boundary value problems. To do so, we will deduce from Corollary 1.4 a recursive procedure which produces at every iteration a better approximation of the solution summing either a slow variable term or the combination of a fast variable and a reflected fast variable term.

\subsection{Structure of the paper}

The paper is organized as follows. In Section 2, we present some preliminary results in potential theory and some technical statements from [1]. In particular, we introduce a fundamental tool in our analysis: the layer potentials with integral kernels consisting of the Dirichlet Green's function of the half-space and of its normal derivative. In Section 3, we prove Theorem 1.3, where we provide a global representation formula in terms of real analytic operators. Section 4 is devoted to the computation of the asymptotic expansion.

\section{Preliminaries}

\subsection{Preliminary of potential theory}

As a first step, we introduce the classical layer potentials for the Laplace equation. We denote by $S_{n}$ the fundamental solution of $\Delta$ defined by

$$
S_{n}(\mathrm{x}) \equiv \frac{1}{(2-n) s_{n}}|\mathrm{x}|^{2-n}, \quad \forall \mathrm{x} \in \mathbb{R}^{n} \backslash\{0\},
$$

where $s_{n}$ is the $(n-1)$-dimensional measure of the boundary of the unit ball in $\mathbb{R}^{n}$. In the sequel, $\mathcal{D}$ is a generic open bounded connected subset of $\mathbb{R}^{n}$ of class $\mathscr{C}^{1, \alpha}$.

Definition 2.1 (Definition of the layer potentials). For any $\phi \in \mathscr{C}^{0, \alpha}(\partial \mathcal{D})$, we define

$$
v_{S_{n}}[\partial \mathcal{D}, \phi](\mathrm{x}) \equiv \int_{\partial \mathcal{D}} \phi(\mathrm{y}) S_{n}(\mathrm{x}-\mathrm{y}) d \sigma_{\mathrm{y}}, \quad \forall \mathrm{x} \in \mathbb{R}^{n},
$$

where $d \sigma$ denotes the area element on $\partial \mathcal{D}$.

The restrictions of $v_{S_{n}}[\partial \mathcal{D}, \phi]$ to $\overline{\mathcal{D}}$ and to $\mathbb{R}^{n} \backslash \mathcal{D}$ are denoted $v_{S_{n}}^{i}[\partial \mathcal{D}, \phi]$ and $v_{S_{n}}^{e}[\partial \mathcal{D}, \phi]$ respectively (the letter ' $i$ ' stands for 'interior' while the letter ' $e$ ' stands for 'exterior').

For any $\psi \in \mathscr{C}^{1, \alpha}(\partial \mathcal{D})$, we define

$$
w_{S_{n}}[\partial \mathcal{D}, \psi](\mathrm{x}) \equiv-\int_{\partial \mathcal{D}} \psi(\mathrm{y}) \mathbf{n}_{\mathcal{D}}(\mathrm{y}) \cdot \nabla S_{n}(\mathrm{x}-\mathrm{y}) d \sigma_{\mathrm{y}}, \quad \forall \mathrm{x} \in \mathbb{R}^{n}
$$

where $\mathbf{n}_{\mathcal{D}}$ denotes the outer unit normal to $\partial \mathcal{D}$ and the symbol $\cdot$ denotes the scalar product in $\mathbb{R}^{n}$.

In Proposition 2.3 here below we recall some classical regularity properties of these layer potentials. Before doing so, we need to introduce the following definition. 
Definition 2.2. We denote by $\mathscr{C}_{\mathrm{loc}}^{1, \alpha}\left(\mathbb{R}^{n} \backslash \mathcal{D}\right)$ the space of functions on $\mathbb{R}^{n} \backslash \mathcal{D}$ whose restrictions to $\overline{\mathcal{O}}$ belong to $\mathscr{C}^{1, \alpha}(\overline{\mathcal{O}})$ for all open bounded subsets $\mathcal{O}$ of $\mathbb{R}^{n} \backslash \mathcal{D}$.

We observe that $\mathscr{C}_{\text {loc }}^{1, \alpha}\left(\mathbb{R}^{n} \backslash \mathcal{D}\right)$ is a Fréchet space with the family of seminorms $\|\cdot\|_{\mathscr{C} 1, \alpha(\overline{\mathcal{O}})}$, with $\mathcal{O} \subseteq \mathbb{R}^{n} \backslash \mathcal{D}$ open and bounded. Then we have the following.

Proposition 2.3 (Regularity of layer potentials). Let $\phi \in \mathscr{C}^{0, \alpha}(\partial \mathcal{D})$ and $\psi \in \mathscr{C}^{1, \alpha}(\partial \mathcal{D})$. Then

- the functions $v_{S_{n}}[\partial \mathcal{D}, \phi]$ and $w_{S_{n}}[\partial \mathcal{D}, \phi]$ are harmonic in $\mathbb{R}^{n} \backslash \mathcal{D}$;

- the function $v_{S_{n}}[\partial \mathcal{D}, \phi]$ is continuous from $\mathbb{R}^{n}$ to $\mathbb{R}$ and the restrictions $v_{S_{n}}^{i}[\partial \mathcal{D}, \phi]$ and $v_{S_{n}}^{e}[\partial \mathcal{D}, \phi]$ belong to $\mathscr{C}^{1, \alpha}(\overline{\mathcal{D}})$ and to $\mathscr{C}_{\text {loc }}^{1, \alpha}\left(\mathbb{R}^{n} \backslash \mathcal{D}\right)$, respectively;

- the restriction $w_{S_{n}}[\partial \mathcal{D}, \psi]_{\mid \mathcal{D}}$ extends to a function $w_{S_{n}}^{i}[\partial \mathcal{D}, \psi]$ of $\mathscr{C}^{1, \alpha}(\overline{\mathcal{D}})$ and the restriction $w_{S_{n}}[\partial \mathcal{D}, \psi]_{\mid \mathbb{R}^{n} \backslash \overline{\mathcal{D}}}$ extends to a function $w_{S_{n}}^{e}[\partial \mathcal{D}, \psi]$ of $\mathscr{C}_{\mathrm{loc}}^{1, \alpha}\left(\mathbb{R}^{n} \backslash \mathcal{D}\right)$.

Moreover,

- the map from $\mathscr{C}^{0, \alpha}(\partial \mathcal{D})$ to $\mathscr{C}^{1, \alpha}(\overline{\mathcal{D}})$ which takes $\phi$ to $v_{S_{n}}^{i}[\partial \mathcal{D}, \phi]$ and the map from $\mathscr{C}^{0, \alpha}(\partial \mathcal{D})$ to $\mathscr{C}_{\text {loc }}^{1, \alpha}\left(\mathbb{R}^{n} \backslash \mathcal{D}\right)$ which takes $\phi$ to $v_{S_{n}}^{e}[\partial \mathcal{D}, \phi]$ are linear and continuous;

- the map from $\mathscr{C}^{1, \alpha}(\partial \mathcal{D})$ to $\mathscr{C}^{1, \alpha}(\overline{\mathcal{D}})$ which takes $\psi$ to $w_{S_{n}}^{i}[\partial \mathcal{D}, \phi]$ and the map from $\mathscr{C}^{1, \alpha}(\partial \mathcal{D})$ to $\mathscr{C}_{\text {loc }}^{1, \alpha}\left(\mathbb{R}^{n} \backslash \mathcal{D}\right)$ which takes $\psi$ to $w_{S_{n}}^{e}[\partial \mathcal{D}, \phi]$ are linear and continuous.

In [1, a key tool for the analysis of problem (1.1) are layer potentials constructed with the Dirichlet Green's function of the upper half-space instead of the classical fundamental solution $S_{n}$. These special layer potentials allow us to transform problem (1.1) into a system of integral equations with no integral equation on $\partial_{0} \Omega$, which is the part of the boundary of $\partial \Omega$ where the inclusion $\omega_{\varepsilon}$ collapses for $\varepsilon=\mathbf{0}$.

Thus we denote by $G$ the Dirichlet Green's function for the upper half-space $\mathbb{R}_{+}^{n}$ defined by

$$
G(\mathrm{x}, \mathrm{y}) \equiv S_{n}(\mathrm{x}-\mathrm{y})-S_{n}(\varsigma(\mathrm{x})-\mathrm{y}), \quad \forall(\mathrm{x}, \mathrm{y}) \in \mathbb{R}^{n} \times \mathbb{R}^{n} \text { with } \mathrm{y} \neq \mathrm{x} \text { and } \mathrm{y} \neq \varsigma(\mathrm{x})
$$

We observe that

$$
G(\mathrm{x}, \mathrm{y})=G(\mathrm{y}, \mathrm{x}), \quad \forall(\mathrm{x}, \mathrm{y}) \in \mathbb{R}^{n} \times \mathbb{R}^{n} \text { with } \mathrm{y} \neq \mathrm{x} \text { and } \mathrm{y} \neq \varsigma(\mathrm{x})
$$

and

$$
G(\mathrm{x}, \mathrm{y})=0, \quad \forall(\mathrm{x}, \mathrm{y}) \in \partial \mathbb{R}_{+}^{n} \times \mathbb{R}^{n} \text { with } \mathrm{y} \neq \mathrm{x} \text { and } \mathrm{y} \neq \varsigma(\mathrm{x}) .
$$

If $\mathcal{D}$ is a subset of $\mathbb{R}^{n}$, we find convenient to set $\varsigma(\mathcal{D}) \equiv\left\{\mathrm{x} \in \mathbb{R}^{n} \mid \varsigma(\mathrm{x}) \in \mathcal{D}\right\}$. We now introduce analogs of the classical layer potentials of Definition 2.1 obtained by replacing $S_{n}$ by the Green's function $G$. In the sequel, $\mathcal{D}_{+}$denotes an open bounded connected set contained in $\mathbb{R}_{+}^{n}$ and of class $\mathscr{C}^{1, \alpha}$.

Definition 2.4 (Definition of layer potentials derived by $G$ ). For any $\phi \in \mathscr{C}^{0, \alpha}\left(\partial \mathcal{D}_{+}\right)$, we define

$$
v_{G}\left[\partial \mathcal{D}_{+}, \phi\right](\mathrm{x}) \equiv \int_{\partial \mathcal{D}_{+}} G(\mathrm{x}, \mathrm{y}) \phi(\mathrm{y}) d \sigma_{\mathrm{y}}, \quad \forall \mathrm{x} \in \mathbb{R}^{n}
$$


The restrictions of $v_{G}\left[\partial \mathcal{D}_{+}, \phi\right]$ to $\overline{\mathcal{D}}_{+}$and $\overline{\mathbb{R}_{+}^{n} \backslash \mathcal{D}_{+}}$are denoted $v_{G}^{i}\left[\partial \mathcal{D}_{+}, \phi\right]$ and $v_{G}^{e}\left[\partial \mathcal{D}_{+}, \phi\right]$ respectively.

For any $\psi \in \mathscr{C}^{1, \alpha}\left(\partial \mathcal{D}_{+}\right)$, we define

$$
w_{G}\left[\partial \mathcal{D}_{+}, \psi\right](\mathrm{x}) \equiv \int_{\partial \mathcal{D}_{+}} \psi(\mathrm{y}) \mathbf{n}_{\mathcal{D}_{+}}(\mathrm{y}) \cdot \nabla_{\mathrm{y}} G(\mathrm{x}, \mathrm{y}) d \sigma_{\mathrm{y}}, \quad \forall \mathrm{x} \in \mathbb{R}^{n} .
$$

To describe the regularity properties of these layer potentials, we shall need the following definition.

Definition 2.5. We denote by $\mathscr{C}_{\text {loc }}^{1, \alpha}\left(\overline{\mathbb{R}_{+}^{n} \backslash \mathcal{D}_{+}}\right)$the space of functions on $\overline{\mathbb{R}_{+}^{n} \backslash \mathcal{D}_{+}}$whose restrictions to $\overline{\mathcal{O}}$ belong to $\mathscr{C}^{1, \alpha}(\overline{\mathcal{O}})$ for all open bounded subsets $\mathcal{O}$ of $\overline{\mathbb{R}_{+}^{n} \backslash \mathcal{D}_{+}}$.

Then we have the following regularity properties that can be deduced from the corresponding properties of classical layer potentials (cf. Proposition 2.3).

Proposition 2.6 (Regularity for the layer potentials derived by $G)$. Let $\phi \in \mathscr{C}^{0, \alpha}\left(\partial \mathcal{D}_{+}\right)$and $\psi \in \mathscr{C}^{1, \alpha}\left(\partial \mathcal{D}_{+}\right)$. Then

- the functions $v_{G}\left[\partial \mathcal{D}_{+}, \phi\right]$ and $w_{G}\left[\partial \mathcal{D}_{+}, \psi\right]$ are harmonic in $\mathcal{D}_{+} \cup \varsigma\left(\mathcal{D}_{+}\right)$, and $\mathbb{R}^{n} \backslash \overline{\mathcal{D}_{+} \cup \varsigma\left(\mathcal{D}_{+}\right)}$;

- the function $v_{G}\left[\partial \mathcal{D}_{+}, \phi\right]$ is continuous from $\mathbb{R}^{n}$ to $\mathbb{R}$ and the restrictions $v_{G}^{i}\left[\partial \mathcal{D}_{+}, \phi\right]$ and $v_{G}^{e}\left[\partial \mathcal{D}_{+}, \phi\right]$ belong to $\mathscr{C}^{1, \alpha}\left(\overline{\mathcal{D}_{+}}\right)$and to $\mathscr{C}_{\mathrm{loc}}^{1, \alpha}\left(\overline{\mathbb{R}_{+}^{n} \backslash \mathcal{D}_{+}}\right)$, respectively;

- the restriction $w_{G}\left[\partial \mathcal{D}_{+}, \psi\right]_{\mid \Omega}$ extends to a function $w_{G}^{i}\left[\partial \mathcal{D}_{+}, \psi\right]$ of $\mathscr{C}^{1, \alpha}\left(\overline{\mathcal{D}_{+}}\right)$and the restriction $w_{G}\left[\partial \mathcal{D}_{+}, \psi\right]_{\mid \mathbb{R}_{+}^{n} \backslash \overline{\mathcal{D}_{+}}}$extends to a function $w_{G}^{e}\left[\partial \mathcal{D}_{+}, \psi\right]$ of $\mathscr{C}_{\mathrm{loc}}^{1, \alpha}\left(\overline{\mathbb{R}_{+}^{n} \backslash \mathcal{D}_{+}}\right)$.

Moreover,

- the map from $\mathscr{C}^{0, \alpha}\left(\partial \mathcal{D}_{+}\right)$to $\mathscr{C}^{1, \alpha}\left(\overline{\mathcal{D}_{+}}\right)$which takes $\phi$ to $v_{G}^{i}\left[\partial \mathcal{D}_{+}, \phi\right]$ and the map from $\mathscr{C}^{0, \alpha}\left(\partial \mathcal{D}_{+}\right)$to $\mathscr{C}_{\mathrm{loc}}^{1, \alpha}\left(\overline{\mathbb{R}_{+}^{n} \backslash \mathcal{D}_{+}}\right)$which takes $\phi$ to $v_{G}^{e}\left[\partial \mathcal{D}_{+}, \phi\right]$ are linear and continuous;

- the map from $\mathscr{C}^{1, \alpha}\left(\partial \mathcal{D}_{+}\right)$to $\mathscr{C}^{1, \alpha}\left(\overline{\mathcal{D}_{+}}\right)$which takes $\psi$ to $w_{G}^{i}\left[\partial \mathcal{D}_{+}, \phi\right]$ and the map from $\mathscr{C}^{1, \alpha}\left(\partial \mathcal{D}_{+}\right)$to $\mathscr{C}_{\text {loc }}^{1, \alpha}\left(\mathbb{R}^{n} \backslash \mathcal{D}_{+}\right)$which takes $\psi$ to $w_{G}^{e}\left[\partial \mathcal{D}_{+}, \phi\right]$ are linear and continuous.

\subsection{Integral representation formulas for $u_{\varepsilon}$}

In [1], to analyze the $\varepsilon$-dependent boundary value problem (1.1), we have exploited the layer potentials with kernel derived by $G$ in the case when $\mathcal{D}=\Omega_{\varepsilon}$. Since $\partial \Omega_{\varepsilon}=\partial \Omega \cup \partial \omega_{\varepsilon}$, we need to consider layer potentials integrated on $\partial \Omega$ and on $\partial \omega_{\varepsilon}$. As one can easily see, the single layer potential $v_{G}[\partial \Omega, \phi]$ does not depend on the values of the density $\phi$ on $\partial_{0} \Omega$, and therefore it is convenient to introduce the quotient Banach space

$$
\mathscr{C}_{+}^{0, \alpha}(\partial \Omega) \equiv \mathscr{C}^{0, \alpha}(\partial \Omega) /\left\{\phi \in \mathscr{C}^{0, \alpha}(\partial \Omega) \mid \phi_{\mid \partial_{+} \Omega}=0\right\} .
$$

One of the key steps in the proof of Theorems 1.1 and 1.2 is the following result, where we prove 'macroscopic' and 'microscopic' representation formulas in terms of integral operators with densities which depend real analytically on the perturbation parameter pair $\varepsilon$ (see [1, Thm. 3.3, Remarks 3.4, 3.5]). 
Theorem 2.7. There exist $0<\varepsilon^{*}<\varepsilon^{\text {ad }}$ and a real analytic map $\mathrm{M} \equiv\left(\mathrm{M}_{1}, \mathrm{M}_{2}\right)$ from ]$-\varepsilon^{*}, \varepsilon^{*}\left[\right.$ to $\mathscr{C}_{+}^{0, \alpha}(\partial \Omega) \times \mathscr{C}^{0, \alpha}(\partial \omega)$ such that the following representation formulas hold:

(i) For all $\varepsilon \in] \mathbf{0}, \varepsilon^{*}[$, we have

$$
\begin{aligned}
u_{\boldsymbol{\varepsilon}}(\mathrm{x})= & u_{\mathbf{0}}(\mathrm{x})-\varepsilon_{1}^{n-1} \varepsilon_{2}^{n-1} \int_{\partial \omega} \mathbf{n}_{\omega}(\mathrm{Y}) \cdot\left(\nabla_{\mathrm{y}} G\right)\left(\mathrm{x}, \varepsilon_{1} \mathrm{p}+\varepsilon_{1} \varepsilon_{2} \mathrm{Y}\right) g^{\mathrm{i}}(\mathrm{Y}) d \sigma_{\mathrm{Y}} \\
& -\int_{\partial_{+} \Omega} G(\mathrm{x}, \mathrm{y}) \mathrm{M}_{1}[\varepsilon](\mathrm{y}) d \sigma_{\mathrm{y}} \\
& +\varepsilon_{1}^{n-2} \varepsilon_{2}^{n-2} \int_{\partial \omega} G\left(\mathrm{x}, \varepsilon_{1} \mathrm{p}+\varepsilon_{1} \varepsilon_{2} \mathrm{Y}\right) \mathrm{M}_{2}[\varepsilon](\mathrm{Y}) d \sigma_{\mathrm{Y}}, \quad \forall \mathrm{x} \in \Omega_{\varepsilon} .
\end{aligned}
$$

(ii) For all $\mathrm{X} \in \mathbb{R}^{n} \backslash \omega$ and all $\left.\varepsilon=\left(\varepsilon_{1}, \varepsilon_{2}\right) \in\right] \mathbf{0}, \varepsilon^{*}\left[\right.$ such that $\varepsilon_{1} \mathrm{p}+\varepsilon_{1} \varepsilon_{2} \mathrm{X} \in \overline{\Omega_{\varepsilon}}$, we have

$$
\begin{aligned}
u_{\boldsymbol{\varepsilon}}\left(\varepsilon_{1} \mathrm{p}+\varepsilon_{1} \varepsilon_{2} \mathrm{X}\right)= & u_{\mathbf{0}}\left(\varepsilon_{1} \mathrm{p}+\varepsilon_{1} \varepsilon_{2} \mathrm{X}\right)-w_{S_{n}}^{e}\left[\partial \omega, g^{\mathrm{i}}\right](\mathbf{X}) \\
& -\varepsilon_{2}^{n-1} \int_{\partial \omega} \mathbf{n}_{\omega}(\mathrm{Y}) \cdot \nabla S_{n}\left(-2 p_{n} \mathrm{e}_{n}+\varepsilon_{2}(\varsigma(\mathrm{X})-\mathrm{Y})\right) g^{\mathrm{i}}(\mathrm{Y}) d \sigma_{\mathbf{Y}} \\
& -\int_{\partial_{+} \Omega} G\left(\varepsilon_{1} \mathrm{p}+\varepsilon_{1} \varepsilon_{2} \mathrm{X}, \mathrm{y}\right) \mathrm{M}_{1}[\varepsilon](\mathrm{y}) d \sigma_{\mathrm{y}} \\
& +v_{S_{n}}\left[\partial \omega, \mathrm{M}_{2}[\varepsilon]\right](\mathbf{X}) \\
& -\varepsilon_{2}^{n-2} \int_{\partial \omega} S_{n}\left(-2 p_{n} \mathrm{e}_{n}+\varepsilon_{2}(\varsigma(\mathbf{X})-\mathrm{Y})\right) \mathrm{M}_{2}[\varepsilon](\mathrm{Y}) d \sigma_{\mathbf{Y}}
\end{aligned}
$$

Here and in what follows, $\mathrm{e}_{n}$ is the vector $(0, \ldots, 0,1)$ of $\mathbb{R}^{n}$.

Moreover, $\mathrm{M}_{1}[\mathbf{0}]=0$ and $\mathrm{M}_{2}[\mathbf{0}]$ is the unique function in $\mathscr{C}^{0, \alpha}(\partial \omega)$ such that

$$
v_{S_{n}}\left[\partial \omega, \mathrm{M}_{2}[\mathbf{0}]\right]_{\mid \partial \omega}=-g^{\mathrm{o}}(0)+w_{S_{n}}\left[\partial \omega, g^{\mathrm{i}}\right]_{\mid \partial \omega}+\frac{g^{\mathrm{i}}}{2} .
$$

\section{Real analytic continuation in global spaces}

\subsection{Existence of the expansion. Proof of Theorem 2.7}

The integral representations of Theorem 2.7 can be used to prove the local analyticity results of Theorems 1.1 and 1.2 . Instead, to obtain the global analyticity result of Theorem 1.3 , we need to modify such representations and express $u_{\varepsilon}$ as a sum of single and double layer potentials. The advantage is that, by virtue of Propositions 2.3 and 2.6 , single and double layer potentials have continuous extensions up to the closure $\overline{\Omega_{\varepsilon}}$ of $\Omega_{\varepsilon}$.

Lemma 3.1. Let $\varepsilon^{*}$ and $\mathrm{M} \equiv\left(\mathrm{M}_{1}, \mathrm{M}_{2}\right)$ be as in Theorem 2.7. Then

$$
\begin{aligned}
& u_{\boldsymbol{\varepsilon}}(\mathrm{x})=u_{\mathbf{0}}(\mathrm{x})-w_{S_{n}}^{e}\left[\partial \omega, g^{\mathrm{i}}\right]\left(\frac{\mathrm{x}-\varepsilon_{1} \mathrm{p}}{\varepsilon_{1} \varepsilon_{2}}\right)+w_{S_{n}}^{e}\left[\partial \omega, g^{\mathrm{i}}\right]\left(\frac{\varsigma(\mathrm{x})-\varepsilon_{1} \mathrm{p}}{\varepsilon_{1} \varepsilon_{2}}\right) \\
& \quad-v_{G}^{i}\left[\partial \Omega, \mathrm{M}_{1}[\varepsilon]\right](\mathrm{x})+v_{S_{n}}^{e}\left[\partial \omega, \mathrm{M}_{2}[\varepsilon]\right]\left(\frac{\mathrm{x}-\varepsilon_{1} \mathrm{p}}{\varepsilon_{1} \varepsilon_{2}}\right)-v_{S_{n}}^{e}\left[\partial \omega, \mathrm{M}_{2}[\varepsilon]\right]\left(\frac{\varsigma(\mathrm{x})-\varepsilon_{1} \mathrm{p}}{\varepsilon_{1} \varepsilon_{2}}\right),
\end{aligned}
$$

for all $\mathrm{x} \in \overline{\Omega_{\varepsilon}}$ and for all $\left.\varepsilon \in\right] \mathbf{0}, \varepsilon^{*}[$. 
Proof. We observe that

$$
G\left(\varepsilon_{1} \mathrm{p}+\varepsilon_{1} \varepsilon_{2} \mathrm{X}, \varepsilon_{1} \mathrm{p}+\varepsilon_{1} \varepsilon_{2} \mathrm{Y}\right)=\varepsilon_{1}^{2-n} \varepsilon_{2}^{2-n} S_{n}(\mathrm{X}-\mathrm{Y})-\varepsilon_{1}^{2-n} \varepsilon_{2}^{2-n} S_{n}\left(\varsigma(\mathrm{X})-\frac{2 p_{n} \mathrm{e}_{n}}{\varepsilon_{2}}-\mathrm{Y}\right)
$$

and

$$
\begin{aligned}
\left(\nabla_{y} G\right)\left(\varepsilon_{1} \mathrm{p}+\varepsilon_{1} \varepsilon_{2} \mathrm{X}, \varepsilon_{1} \mathrm{p}\right. & \left.+\varepsilon_{1} \varepsilon_{2} \mathrm{Y}\right) \\
= & \varepsilon_{1}^{1-n} \varepsilon_{2}^{1-n}\left(\nabla S_{n}\right)\left(\varsigma(\mathrm{X})-\frac{2 p_{n} \mathrm{e}_{n}}{\varepsilon_{2}}-\mathrm{Y}\right)-\varepsilon_{1}^{1-n} \varepsilon_{2}^{1-n}\left(\nabla S_{n}\right)(\mathrm{X}-\mathrm{Y}),
\end{aligned}
$$

for all $\mathrm{X}, \mathrm{Y} \in \mathbb{R}^{n}$ and all $\boldsymbol{\varepsilon}>\mathbf{0}$ such that $\varepsilon_{1} \mathrm{p}+\varepsilon_{1} \varepsilon_{2} \mathrm{X} \neq \varepsilon_{1} \mathrm{p}+\varepsilon_{1} \varepsilon_{2} \mathrm{Y}$ and $\varsigma\left(\varepsilon_{1} \mathrm{p}+\varepsilon_{1} \varepsilon_{2} \mathrm{X}\right) \neq$ $\varepsilon_{1} \mathrm{p}+\varepsilon_{1} \varepsilon_{2} \mathrm{Y}$ (or, equivalently, such that $\mathrm{X} \neq \mathrm{Y}$ and $\varsigma(\mathrm{X}) \neq \frac{2 p_{n} \mathrm{e}_{n}}{\varepsilon_{2}}+\mathrm{Y}$ ). Then, taking

$$
x=\varepsilon_{1} p+\varepsilon_{1} \varepsilon_{2}\left(\frac{x-\varepsilon_{1} p}{\varepsilon_{1} \varepsilon_{2}}\right)
$$

in (2.1) we deduce that

$$
\begin{aligned}
u_{\boldsymbol{\varepsilon}}(\mathrm{x})= & u_{\mathbf{0}}(\mathrm{x})+\int_{\partial \omega} \mathbf{n}_{\omega}(\mathrm{Y}) \cdot\left(\nabla S_{n}\right)\left(\left(\frac{\mathrm{x}-\varepsilon_{1} \mathrm{p}}{\varepsilon_{1} \varepsilon_{2}}\right)-\mathrm{Y}\right) g^{\mathrm{i}}(\mathrm{Y}) d \sigma_{\mathrm{Y}} \\
& -\int_{\partial \omega} \mathbf{n}_{\omega}(\mathrm{Y}) \cdot\left(\nabla S_{n}\right)\left(\varsigma\left(\frac{\mathrm{x}-\varepsilon_{1} \mathrm{p}}{\varepsilon_{1} \varepsilon_{2}}\right)-\frac{2 p_{n} \mathrm{e}_{n}}{\varepsilon_{2}}-\mathrm{Y}\right) g^{\mathrm{i}}(\mathrm{Y}) d \sigma_{\mathrm{Y}} \\
& -\int_{\partial_{+} \Omega} G(\mathrm{x}, \mathrm{y}) \mathrm{M}_{1}[\varepsilon](\mathrm{y}) d \sigma_{\mathrm{y}} \\
& +\int_{\partial \omega} S_{n}\left(\left(\frac{\mathrm{x}-\varepsilon_{1} \mathrm{p}}{\varepsilon_{1} \varepsilon_{2}}\right)-\mathrm{Y}\right) \mathrm{M}_{2}[\varepsilon](\mathrm{Y}) d \sigma_{\mathrm{Y}} \\
& -\int_{\partial \omega} S_{n}\left(\varsigma\left(\frac{\mathrm{x}-\varepsilon_{1} \mathrm{p}}{\varepsilon_{1} \varepsilon_{2}}\right)-\frac{2 p_{n} \mathrm{e}_{n}}{\varepsilon_{2}}-\mathrm{Y}\right) \mathrm{M}_{2}[\varepsilon](\mathrm{Y}) d \sigma_{\mathrm{Y}}
\end{aligned}
$$

for all $\varepsilon \in] \mathbf{0}, \varepsilon^{*}\left[\right.$ and $x \in \Omega_{\varepsilon}$. By the definitions of single and double layer potentials for $S_{n}$ and $G$ (cf. Definitions 2.1 and 2.4) it follows that

$$
\begin{aligned}
& u_{\boldsymbol{\varepsilon}}(\mathrm{x})=u_{\mathbf{0}}(\mathrm{x})-w_{S_{n}}\left[\partial \omega, g^{\mathrm{i}}\right]\left(\frac{\mathrm{x}-\varepsilon_{1} \mathrm{p}}{\varepsilon_{1} \varepsilon_{2}}\right)+w_{S_{n}}\left[\partial \omega, g^{\mathrm{i}}\right]\left(\varsigma\left(\frac{\mathrm{x}-\varepsilon_{1} \mathrm{p}}{\varepsilon_{1} \varepsilon_{2}}\right)-\frac{2 p_{n} \mathrm{e}_{n}}{\varepsilon_{2}}\right) \\
& -v_{G}\left[\partial \Omega, \mathrm{M}_{1}[\varepsilon]\right](\mathrm{x})+v_{S_{n}}\left[\partial \omega, \mathrm{M}_{2}[\varepsilon]\right]\left(\frac{\mathrm{x}-\varepsilon_{1} \mathrm{p}}{\varepsilon_{1} \varepsilon_{2}}\right)-v_{S_{n}}\left[\partial \omega, \mathrm{M}_{2}[\varepsilon]\right]\left(\varsigma\left(\frac{\mathrm{x}-\varepsilon_{1} \mathrm{p}}{\varepsilon_{1} \varepsilon_{2}}\right)-\frac{2 p_{n} \mathrm{e}_{n}}{\varepsilon_{2}}\right),
\end{aligned}
$$

for all $\varepsilon \in] \mathbf{0}, \varepsilon^{*}\left[\right.$ and $x \in \Omega_{\varepsilon}$. Then the statement of the lemma is a consequence of equality

$$
\varsigma\left(\frac{\mathrm{x}-\varepsilon_{1} \mathrm{p}}{\varepsilon_{1} \varepsilon_{2}}\right)-\frac{2 p_{n} \mathrm{e}_{n}}{\varepsilon_{2}}=\frac{\varsigma(\mathrm{x})-\varepsilon_{1} \mathrm{p}}{\varepsilon_{1} \varepsilon_{2}},
$$

of the membership of $u_{\varepsilon}$ in $\mathscr{C}^{1, \alpha}\left(\overline{\Omega_{\varepsilon}}\right)$, and of Propositions 2.3 and 2.6 .

We are now ready to prove Theorem 1.3 . 
Proof of Theorem 1.3. We define

$$
\mathfrak{U}[\varepsilon](\mathrm{x}) \equiv u_{\mathbf{0}}(\mathrm{x})-v_{G}^{i}\left[\partial \Omega, \mathrm{M}_{1}[\varepsilon]\right](\mathrm{x}), \quad \forall \mathrm{x} \in \bar{\Omega},
$$

and

$$
\mathfrak{V}[\varepsilon](\mathbf{X}) \equiv-w_{S_{n}}^{e}\left[\partial \omega, g^{\mathrm{i}}\right](\mathbf{X})+v_{S_{n}}^{e}\left[\partial \omega, \mathrm{M}_{2}[\varepsilon]\right](\mathbf{X}), \quad \forall \mathbf{X} \in \mathbb{R}^{n} \backslash \omega,
$$

for all $\varepsilon \in]-\varepsilon^{*}, \varepsilon^{*}[$.

We claim that $\mathfrak{U}$ and $\mathfrak{V}$ are real analytic. Indeed, by Proposition $2.3, v_{G}^{i}[\partial \Omega, \cdot]$ is linear and continuous, and therefore real analytic, from $\mathscr{C}^{0, \alpha}(\partial \Omega)$ to $\mathscr{C}_{\mathrm{h}}^{1, \alpha}(\bar{\Omega})$ and $\mathrm{M}_{1}$ is real analytic from $]-\varepsilon^{*}, \varepsilon^{*}\left[\right.$ to $\mathscr{C}^{0, \alpha}(\partial \Omega)$ by Theorem 2.7. Since the composition of real analytic maps is real analytic it follows that $\mathfrak{U}$ is real analytic from $]-\varepsilon^{*}, \varepsilon^{*}\left[\right.$ to $\mathscr{C}_{\mathrm{h}}^{1, \alpha}(\bar{\Omega})$. To prove that $\mathfrak{V}$ is real analytic we first recall that the map which takes a function $v$ to its trace $v_{\mid \partial \omega}$ is a linear isomorphism between $\mathscr{C}_{\mathrm{h}}^{1, \alpha}\left(\mathbb{R}^{n} \backslash \omega\right)$ and $\mathscr{C}^{1, \alpha}(\partial \omega)$. Since $v_{S_{n}}^{e}[\partial \omega, \phi]$ belongs to $\mathscr{C}_{\mathrm{h}}^{1, \alpha}\left(\mathbb{R}^{n} \backslash \omega\right)$ for all $\phi \in \mathscr{C}^{0, \alpha}(\partial \omega)$ and the map which takes $\phi \in \mathscr{C}^{0, \alpha}(\partial \omega)$ to the trace $v_{S_{n}}^{e}[\partial \omega, \phi]_{\mid \partial \omega}$ is linear and continuous (cf. Proposition 2.3), it follows that the map which takes $\phi$ to $v_{S_{n}}^{e}[\partial \omega, \phi]$ is linear and continuous, and therefore real analytic, from $\mathscr{C}^{0, \alpha}(\partial \omega)$ to $\mathscr{C}_{\mathrm{h}}^{1, \alpha}\left(\mathbb{R}^{n} \backslash \omega\right)$. Since $\mathrm{M}_{2}$ is real analytic from ] $-\varepsilon^{*}, \varepsilon^{*}\left[\right.$ to $\mathscr{C}^{0, \alpha}(\partial \omega)$ (cf. Theorem 2.7) and the composition of real analytic maps is real analytic, we deduce that also $\mathfrak{V}$ is real analytic from $]-\varepsilon^{*}, \varepsilon^{*}\left[\right.$ to $\mathscr{C}_{\mathrm{h}}^{1, \alpha}\left(\mathbb{R}^{n} \backslash \omega\right)$.

The validity of (1.5) follows by Lemma 3.1 and the first equality in 1.6 can be deduced by Theorem 2.7 and by a straightforward computation. To prove the second equality in (1.6) we observe that:

$$
\mathfrak{V}[\mathbf{0}]=-w_{S_{n}}^{e}\left[\partial \omega, g^{\mathrm{i}}\right]+v_{S_{n}}^{e}\left[\partial \omega, \mathrm{M}_{2}[\mathbf{0}]\right]
$$

by Theorem 2.7. Then, by Theorem 2.7 and by the jump properties of the double layer potential (cf., e.g., [1, Prop. 2.4]) we deduce that the right hand side of (3.1) equals $g^{\mathrm{i}}-g^{\mathrm{o}}(0)$ on $\partial \omega$. The second relation of (1.6) follows by the decaying properties at $\infty$ of the single and double layer potentials and by the uniqueness of the solution of the exterior Dirichlet problem.

Finally, to verify that $\mathfrak{U}$ and $\mathfrak{V}$ are unique, we observe that if we have two functions $\phi \in \mathscr{C}_{\mathrm{h}}^{1, \alpha}(\bar{\Omega})$ and $\psi \in \mathscr{C}_{\mathrm{h}}^{1, \alpha}\left(\mathbb{R}^{n} \backslash \omega\right)$ such that

$$
\phi(\mathrm{x})+\psi\left(\frac{\mathrm{x}-\varepsilon_{1} \mathrm{p}}{\varepsilon_{1} \varepsilon_{2}}\right)-\psi\left(\frac{\varsigma(\mathrm{x})-\varepsilon_{1} \mathrm{p}}{\varepsilon_{1} \varepsilon_{2}}\right)=0, \quad \forall \mathrm{x} \in \overline{\Omega_{\varepsilon}}
$$

for some $\varepsilon \in] \mathbf{0}, \varepsilon^{*}[$, then

$$
\psi\left(\frac{\mathrm{x}-\varepsilon_{1} \mathrm{p}}{\varepsilon_{1} \varepsilon_{2}}\right)=-\phi(\mathrm{x})+\psi\left(\frac{\varsigma(\mathrm{x})-\varepsilon_{1} \mathrm{p}}{\varepsilon_{1} \varepsilon_{2}}\right), \quad \forall \mathrm{x} \in \overline{\Omega_{\varepsilon}}
$$

and thus $\psi\left(\frac{\cdot-\varepsilon_{1} p}{\varepsilon_{1} \varepsilon_{2}}\right)$ has an harmonic extension on the whole of $\mathbb{R}^{n}$. Since $\lim _{x \rightarrow \infty} \psi\left(\frac{x-\varepsilon_{1} p}{\varepsilon_{1} \varepsilon_{2}}\right)=$ 0 , the Liouville theorem for harmonic functions implies that $\psi=0$. Then, $\phi=0$ by (3.2) and by the identity principle.

\subsection{On the uniqueness of such a writing}

As we have already observed, Theorem 1.3 implies the validity of an expansion as in 1.8 for $u_{\varepsilon}$. In Proposition 3.2 below, we show a uniqueness result for the coefficients of such an expansion. In what follows we denote by $\mathscr{C}(\bar{\Omega})$ the space of continuous functions on $\bar{\Omega}$ and by $\mathscr{C}_{0}\left(\mathbb{R}^{n} \backslash \omega\right)$ the space of continuous functions on $\mathbb{R}^{n} \backslash \omega$ which vanish at infinity. 
Proposition 3.2. Let $N \in \mathbb{N}$. Let $u_{i, j} \in \mathscr{C}(\bar{\Omega})$ and $v_{i, j} \in \mathscr{C}_{0}\left(\mathbb{R}^{n} \backslash \omega\right)$ for all $(i, j) \in \mathbb{N}^{2}$ with $i+j \leq N$. We denote by $W_{\varepsilon, N}$ the function

$$
W_{\varepsilon, N}(\mathrm{x}) \equiv \sum_{i+j \leq N} \varepsilon_{1}^{i} \varepsilon_{2}^{j} u_{i, j}(\mathrm{x})+\sum_{i+j \leq N} \varepsilon_{1}^{i} \varepsilon_{2}^{j} v_{i, j}\left(\frac{\mathrm{x}-\varepsilon_{1} \mathrm{p}}{\varepsilon_{1} \varepsilon_{2}}\right)-\sum_{i+j \leq N} \varepsilon_{1}^{i} \varepsilon_{2}^{j} v_{i, j}\left(\frac{\varsigma(\mathrm{x})-\varepsilon_{1} \mathrm{p}}{\varepsilon_{1} \varepsilon_{2}}\right)
$$

of $\mathrm{x} \in \overline{\Omega_{\varepsilon}}$. If

$$
\sup _{\mathrm{x} \in \Omega_{\varepsilon}}\left|u_{\varepsilon}(\mathbf{x})-W_{\varepsilon, N}(\mathbf{x})\right|=o\left(\sum_{i+j=N} \varepsilon_{1}^{i} \varepsilon_{2}^{j}\right) \quad \text { as } \boldsymbol{\varepsilon} \rightarrow \mathbf{0},
$$

then $u_{i, j}=U_{i, j}$ and $v_{i, j}=V_{i, j}$ for all $(i, j) \in \mathbb{N}^{2}$ with $i+j \leq N$, where $U_{i, j}, V_{i, j}$ are defined by (1.7).

Proof. For $0<\eta_{*}<1$ sufficiently small we consider the curve in $] \mathbf{0}, \boldsymbol{\varepsilon}^{*}$. which takes $\left.\eta \in\right] 0, \eta_{*}[$ to $\varepsilon(\eta)=\left(\varepsilon_{1}(\eta), \varepsilon_{2}(\eta)\right) \equiv(\eta,-1 / \log \eta)$. Then we observe that $\varepsilon_{1}^{i}(\eta) \varepsilon_{2}^{j}(\eta)=o\left(\varepsilon_{1}^{h}(\eta) \varepsilon_{2}^{k}(\eta)\right)$ as $\eta \rightarrow 0$ if and only if either one of the following conditions holds true:

$$
i>h \quad \text { or } \quad i=h \text { and } j>k \text {. }
$$

Accordingly, we endow $\mathbb{N}^{2}$ with a total order relation by saying that $(i, j)>(h, k)$ if and only if (3.4) is verified, and, as usual, $(i, j) \geq(h, k)$ if $(i, j)=(h, k)$ or $(i, j)>(h, k)$. An elementary argument shows that every subset of $\mathbb{N}^{2}$ has a minimum with respect to $\geq$. Therefore, we are in the position to prove the lemma by an induction argument on $(i, j)$.

We first prove that $u_{0,0}=U_{0,0}$. We note that by the membership of $v_{i, j}$ in $\mathscr{C}_{0}\left(\mathbb{R}^{n} \backslash \omega\right)$ we have

$$
\lim _{\eta \rightarrow 0} v_{i, j}\left(\frac{\mathrm{x}-\varepsilon_{1}(\eta) \mathrm{p}}{\varepsilon_{1}(\eta) \varepsilon_{2}(\eta)}\right)=0 \quad \text { and } \quad \lim _{\eta \rightarrow 0} v_{i, j}\left(\frac{\varsigma(\mathrm{x})-\varepsilon_{1}(\eta) \mathrm{p}}{\varepsilon_{1}(\eta) \varepsilon_{2}(\eta)}\right)=0 \quad \forall \mathrm{x} \in \bar{\Omega}
$$

for all $(i, j) \in \mathbb{N}^{2}$ with $i+j \leq N$. Similarly, by the membership of $V_{i, j}$ in $\mathscr{C}_{\mathrm{h}}^{1, \alpha}\left(\mathbb{R}^{n} \backslash \omega\right)$ we have

$$
\lim _{\eta \rightarrow 0} V_{i, j}\left(\frac{\mathrm{x}-\varepsilon_{1}(\eta) \mathrm{p}}{\varepsilon_{1}(\eta) \varepsilon_{2}(\eta)}\right)=0 \quad \text { and } \quad \lim _{\eta \rightarrow 0} V_{i, j}\left(\frac{\varsigma(\mathrm{x})-\varepsilon_{1}(\eta) \mathrm{p}}{\varepsilon_{1}(\eta) \varepsilon_{2}(\eta)}\right)=0 \quad \forall \mathrm{x} \in \bar{\Omega},
$$

for all $(i, j) \in \mathbb{N}^{2}$. Then, by $(3.5)$ we compute that

$$
\lim _{\eta \rightarrow 0} W_{\varepsilon(\eta), N}(\mathrm{x})=u_{0,0}(\mathrm{x}) \quad \forall \mathrm{x} \in \bar{\Omega},
$$

and, by (1.7) and (3.6) we also have

$$
\lim _{\eta \rightarrow 0} u_{\varepsilon(\eta)}(\mathrm{x})=U_{0,0}(\mathrm{x}) \quad \forall \mathrm{x} \in \bar{\Omega} .
$$

By (3.3) it follows that $u_{0,0}=U_{0,0}$.

To prove that $v_{0,0}=V_{0,0}$, we observe that

$$
\lim _{\eta \rightarrow 0} v_{i, j}\left(\frac{\varsigma\left(\varepsilon_{1}(\eta) \mathrm{p}+\varepsilon_{1}(\eta) \varepsilon_{2}(\eta) \mathrm{X}\right)-\varepsilon_{1}(\eta) \mathrm{p}}{\varepsilon_{1}(\eta) \varepsilon_{2}(\eta)}\right)=\lim _{\eta \rightarrow 0} v_{i, j}\left(\varsigma(\mathrm{X})-\frac{2 p_{n} \mathrm{e}_{n}}{\varepsilon_{2}(\eta)}\right)=0 \quad \forall \mathrm{X} \in \mathbb{R}^{n} \backslash \omega,
$$


for all $(i, j) \in \mathbb{N}^{2}$ with $i+j \leq N$, and similarly

$$
\lim _{\eta \rightarrow 0} V_{i, j}\left(\frac{\varsigma\left(\varepsilon_{1}(\eta) \mathrm{p}+\varepsilon_{1}(\eta) \varepsilon_{2}(\eta) \mathrm{X}\right)-\varepsilon_{1}(\eta) \mathrm{p}}{\varepsilon_{1}(\eta) \varepsilon_{2}(\eta)}\right)=\lim _{\eta \rightarrow 0} V_{i, j}\left(\varsigma(\mathrm{X})-\frac{2 p_{n} \mathrm{e}_{n}}{\varepsilon_{2}(\eta)}\right)=0 \quad \forall \mathbf{X} \in \mathbb{R}^{n} \backslash \omega,
$$

for all $(i, j) \in \mathbb{N}^{2}$. It follows that

$$
\lim _{\eta \rightarrow 0} W_{\varepsilon(\eta), N}\left(\varepsilon_{1}(\eta) \mathrm{p}+\varepsilon_{1}(\eta) \varepsilon_{2}(\eta) \mathbf{X}\right)=u_{0,0}(0)+v_{0,0}(\mathbf{X}) \quad \forall \mathbf{X} \in \mathbb{R}^{n} \backslash \omega,
$$

and that

$$
\lim _{\eta \rightarrow 0} u_{\varepsilon}\left(\varepsilon_{1}(\eta) \mathrm{p}+\varepsilon_{1}(\eta) \varepsilon_{2}(\eta) \mathbf{X}\right)=U_{0,0}(0)+V_{0,0}(\mathbf{X}) \quad \forall \mathbf{X} \in \mathbb{R}^{n} \backslash \omega
$$

(see also (1.7)). Then we observe that 3.3 is equivalent to

$$
\sup _{\mathbf{X} \in\left(\Omega^{\varepsilon} \backslash \bar{\omega}\right)}\left|u_{\varepsilon}\left(\varepsilon_{1} \mathrm{p}+\varepsilon_{1} \varepsilon_{2} \mathbf{X}\right)-W_{\varepsilon, N}\left(\varepsilon_{1} \mathrm{p}+\varepsilon_{1} \varepsilon_{2} \mathbf{X}\right)\right|=o\left(\sum_{i+j=N} \varepsilon_{1}^{i} \varepsilon_{2}^{j}\right) \quad \text { as } \boldsymbol{\varepsilon} \rightarrow \mathbf{0},
$$

where $\Omega^{\varepsilon} \equiv\left(\Omega-\varepsilon_{1} p\right) / \varepsilon_{1} \varepsilon_{2}$. By $(3.9)$ and $(3.10)$ and by equality $u_{0,0}(0)=U_{0,0}(0)$, it follows that $v_{0,0}=V_{0,0}$.

If $N=0$ the proof is complete. We now assume that $N>0$ and that $u_{i, j}=U_{i, j}$ and $v_{i, j}=V_{i, j}$ for all $(i, j) \leq\left(i_{*}, j_{*}\right)$, where $\left(i_{*}, j_{*}\right)$ is a multi-index with $i_{*}+j_{*} \leq N$ and $i_{*}<N$ (note that $(N, 0)$ is the 'biggest' multi-index which appears in the sums of (3.3)). For all $\varepsilon \in] \mathbf{0}, \boldsymbol{\varepsilon}^{*}\left[\right.$ we denote by $W_{\varepsilon,\left(i_{*}, j_{*}\right)}$ the function of $\mathrm{x} \in \Omega_{\varepsilon}$ defined by

$$
\begin{aligned}
& W_{\varepsilon,\left(i_{*}, j_{*}\right)}(\mathrm{x}) \\
& \equiv \sum_{(i, j) \leq\left(i_{*}, j_{*}\right)} \varepsilon_{1}^{i} \varepsilon_{2}^{j} u_{i, j}(\mathrm{x})+\sum_{(i, j) \leq\left(i_{*}, j_{*}\right)} \varepsilon_{1}^{i} \varepsilon_{2}^{j} v_{i, j}\left(\frac{\mathrm{x}-\varepsilon_{1} \mathrm{p}}{\varepsilon_{1} \varepsilon_{2}}\right)-\sum_{(i, j) \leq\left(i_{*}, j_{*}\right)} \varepsilon_{1}^{i} \varepsilon_{2}^{j} v_{i, j}\left(\frac{\varsigma(\mathrm{x})-\varepsilon_{1} \mathrm{p}}{\varepsilon_{1} \varepsilon_{2}}\right),
\end{aligned}
$$

for all $x \in \overline{\Omega_{\varepsilon}}$. Then we denote by $\left(i^{*}, j^{*}\right)$ the minimum of the multi-indexes $(i, j)$ with $i+j \leq N$ which are strictly bigger than $\left(i_{*}, j_{*}\right)$. By (3.5) we compute that

$$
\lim _{\eta \rightarrow 0} \frac{W_{\varepsilon(\eta), N}(\mathrm{x})-W_{\varepsilon(\eta),\left(i_{*}, j_{*}\right)}(\mathrm{x})}{\varepsilon_{1}^{i^{*}}(\eta) \varepsilon_{2}^{j^{*}}(\eta)}=u_{i^{*}, j^{*}}(\mathrm{x}) \quad \forall \mathrm{x} \in \bar{\Omega},
$$

and by (1.7) and (3.6) we have

$$
\lim _{\eta \rightarrow 0} \frac{u_{\varepsilon(\eta)}(\mathrm{x})-W_{\varepsilon(\eta),\left(i_{*}, j_{*}\right)}(\mathrm{x})}{\varepsilon_{1}^{i^{*}}(\eta) \varepsilon_{2}^{j^{*}}(\eta)}=U_{i^{*}, j^{*}}(\mathrm{x}) \quad \forall \mathrm{x} \in \bar{\Omega} .
$$

Accordingly, (3.3) implies that $u_{i^{*}, j^{*}}=U_{i^{*}, j^{*}}$. To show that $v_{i^{*}, j^{*}}=V_{i^{*}, j^{*}}$ we use (3.7) to verify that

$\lim _{\eta \rightarrow 0} \frac{W_{\boldsymbol{\varepsilon}(\eta), N}\left(\varepsilon_{1}(\eta) \mathrm{p}+\varepsilon_{1}(\eta) \varepsilon_{2}(\eta) \mathbf{X}\right)-W_{\boldsymbol{\varepsilon}(\eta),\left(i_{*}, j_{*}\right)}\left(\varepsilon_{1}(\eta) \mathrm{p}+\varepsilon_{1}(\eta) \varepsilon_{2}(\eta) \mathbf{X}\right)}{\varepsilon_{1}^{i^{*}}(\eta) \varepsilon_{2}^{j^{*}}(\eta)}=u_{i^{*}, j^{*}}(0)+v_{i^{*}, j^{*}}(\mathbf{X})$

for all $X \in \mathbb{R}^{n} \backslash \omega$. Similarly, by (1.7) and (3.8) we deduce that

$$
\lim _{\eta \rightarrow 0} \frac{u_{\varepsilon(\eta)}\left(\varepsilon_{1}(\eta) \mathrm{p}+\varepsilon_{1}(\eta) \varepsilon_{2}(\eta) \mathrm{X}\right)-W_{\boldsymbol{\varepsilon}(\eta),\left(i_{*}, j_{*}\right)}\left(\varepsilon_{1}(\eta) \mathrm{p}+\varepsilon_{1}(\eta) \varepsilon_{2}(\eta) \mathbf{X}\right)}{\varepsilon_{1}^{i^{*}}(\eta) \varepsilon_{2}^{j^{*}}(\eta)}=U_{i^{*}, j^{*}}(0)+V_{i^{*}, j^{*}}(\mathbf{X})
$$

for all $\mathrm{X} \in \mathbb{R}^{n} \backslash \omega$. Then (3.11) and equality $u_{i^{*}, j^{*}}(0)=U_{i^{*}, j^{*}}(0)$ imply that $v_{i^{*}, j^{*}}=V_{i^{*}, j^{*}}$. Our proof is now complete. 


\subsection{On the necessity of the reflected terms.}

Proposition 3.2 shows that the solution $u_{\varepsilon}$ can be approximated by a finite sum of slow variable terms, fast variable terms, and reflected fast variable terms. In the following Remark 3.3 we investigate the possibility of approximating the solution $u_{\varepsilon}$ by a finite sum of slow variable and fast variable terms, but no reflected fast variable terms. By means of an example in $\mathbb{R}^{3}$, we prove that in general this is not possible, at least not if we want the terms in the sum to be continuous functions.

Example 3.3. Let $n=3$ and assume that $g^{\mathrm{i}}$ and $g^{\mathrm{o}}$ are such that $v_{\mathbf{0}}(\mathrm{X})=1 /|\mathrm{X}|$ for all $\mathrm{X} \in \mathbb{R}^{3} \backslash \omega$ (cf. problem (1.4)). Then, there are no functions $u_{0,0}^{\#}, u_{0,1}^{\#}, u_{1,0}^{\#} \in \mathscr{C}(\bar{\Omega})$ and $v_{0,0}^{\#}, v_{0,1}^{\#}, v_{1,0}^{\#} \in \mathscr{C}_{0}\left(\mathbb{R}^{3} \backslash \omega\right)$ such that

$$
\sup _{\mathrm{x} \in \Omega_{\varepsilon}}\left|u_{\varepsilon}(\mathrm{x})-W_{\varepsilon, 1}^{\#}(\mathrm{x})\right|=o\left(\varepsilon_{1}+\varepsilon_{2}\right) \quad \text { as } \boldsymbol{\varepsilon} \rightarrow \mathbf{0},
$$

where

$$
\begin{aligned}
& W_{\varepsilon, 1}^{\#}(\mathrm{x}) \\
& \equiv u_{0,0}^{\#}(\mathrm{x})+v_{0,0}^{\#}\left(\frac{\mathrm{x}-\varepsilon_{1} \mathrm{p}}{\varepsilon_{1} \varepsilon_{2}}\right)+\varepsilon_{2} u_{0,1}^{\#}(\mathrm{x})+\varepsilon_{2} v_{0,1}^{\#}\left(\frac{\mathrm{x}-\varepsilon_{1} \mathrm{p}}{\varepsilon_{1} \varepsilon_{2}}\right)+\varepsilon_{1} u_{1,0}^{\#}(\mathrm{x})+\varepsilon_{1} v_{1,0}^{\#}\left(\frac{\mathrm{x}-\varepsilon_{1} \mathrm{p}}{\varepsilon_{1} \varepsilon_{2}}\right)
\end{aligned}
$$

for all $\mathrm{x} \in \overline{\Omega_{\varepsilon}}$.

Proof. We assume by contradiction that there exist $u_{0,0}^{\#}, u_{0,1}^{\#}, u_{1,0}^{\#} \in \mathscr{C}(\bar{\Omega})$ and $v_{0,0}^{\#}, v_{0,1}^{\#}, v_{1,0}^{\#} \in$ $\mathscr{C}_{0}\left(\mathbb{R}^{3} \backslash \omega\right)$ such that $(3.12$ holds true. As in the proof of Proposition 3.2 , we take $0<$ $\eta_{*}<1$ small enough and we consider the curve in $] \mathbf{0}, \varepsilon^{*}[$ which takes $\eta \in] 0, \eta_{*}[$ to $\varepsilon(\eta)=$ $\left(\varepsilon_{1}(\eta), \varepsilon_{2}(\eta)\right) \equiv(\eta,-1 / \log \eta)$. By the membership of $v_{0,0}^{\#}, v_{0,1}^{\#}, v_{1,0}^{\#}$ in $\mathscr{C}_{0}\left(\mathbb{R}^{3} \backslash \omega\right)$ we have

$$
\lim _{\eta \rightarrow 0} v_{i, j}^{\#}\left(\frac{\mathrm{x}-\varepsilon_{1}(\eta) \mathrm{p}}{\varepsilon_{1}(\eta) \varepsilon_{2}(\eta)}\right)=0 \quad \forall \mathrm{x} \in \bar{\Omega},(i, j) \in\{(0,0),(0,1),(1,0)\} .
$$

Then, by arguing as we have done in the proof of Proposition 3.2 to show that $u_{0,0}=U_{0,0}$ and $v_{0,0}=V_{0,0}$, we can verify that $u_{0,0}^{\#}=U_{0,0}$ and $v_{0,0}^{\#}=V_{0,0}$. In particular, $u_{0,0}^{\#}=u_{0}$ and $v_{0,0}^{\#}(\mathbf{X})=v_{\mathbf{0}}(\mathbf{X})=1 /|\mathbf{X}|$ for all $\mathbf{X} \in \mathbb{R}^{3} \backslash \omega\left(\right.$ cf. Theorem 1.3. Since $\varepsilon_{1}(\eta)=o\left(\varepsilon_{2}(\eta)\right)$ as $\eta \rightarrow 0$ the asymptotic relation $(3.12)$ and the limit 3.13 imply that

$$
\lim _{\eta \rightarrow 0} \frac{1}{\varepsilon_{2}(\eta)}\left(u_{\varepsilon}(\mathrm{x})-W_{\varepsilon(\eta),(0,1)}^{\#}(\mathrm{x})\right)=0 \quad \forall \mathrm{x} \in \bar{\Omega}
$$

where

$$
W_{\boldsymbol{\varepsilon}(\eta),(0,1)}^{\#}(\mathrm{x}) \equiv u_{\mathbf{0}}(\mathrm{x})+v_{\mathbf{0}}\left(\frac{\mathrm{x}-\varepsilon_{1}(\eta) \mathrm{p}}{\varepsilon_{1}(\eta) \varepsilon_{2}(\eta)}\right)+\varepsilon_{2}(\eta) u_{0,1}^{\#}(\mathrm{x})+\varepsilon_{2}(\eta) v_{0,1}^{\#}\left(\frac{\mathrm{x}-\varepsilon_{1}(\eta) \mathrm{p}}{\varepsilon_{1}(\eta) \varepsilon_{2}(\eta)}\right),
$$

for all $x \in \overline{\Omega_{\varepsilon(\eta)}}$. By $(3.13$ we deduce that

$$
\lim _{\eta \rightarrow 0} \frac{1}{\varepsilon_{2}(\eta)}\left(W_{\boldsymbol{\varepsilon}(\eta),(0,1)}^{\#}(\mathrm{x})-u_{\mathbf{0}}(\mathrm{x})-v_{\mathbf{0}}\left(\frac{\mathrm{x}-\varepsilon_{1}(\eta) \mathrm{p}}{\varepsilon_{1}(\eta) \varepsilon_{2}(\eta)}\right)\right)=u_{0,1}^{\#}(\mathrm{x}) \quad \forall \mathrm{x} \in \bar{\Omega} .
$$


Then we observe that

$$
\lim _{\eta \rightarrow 0} \frac{1}{\varepsilon_{2}(\eta)} v_{\mathbf{0}}\left(\frac{\varsigma(\mathrm{x})-\varepsilon_{1}(\eta) \mathrm{p}}{\varepsilon_{1}(\eta) \varepsilon_{2}(\eta)}\right)=\lim _{\eta \rightarrow 0} \frac{\varepsilon_{1}(\eta)}{\left|\varsigma(\mathrm{x})-\varepsilon_{1}(\eta) \mathrm{p}\right|}=0 \quad \forall \mathrm{x} \in \bar{\Omega} \backslash\{0\}
$$

(note that we cannot take $x=0$ in this limit relation). Therefore we deduce by (1.7) and (3.6) that

$$
\lim _{\eta \rightarrow 0} \frac{1}{\varepsilon_{2}(\eta)}\left(u_{\varepsilon(\eta)}(\mathrm{x})-u_{\mathbf{0}}(\mathrm{x})-v_{\mathbf{0}}\left(\frac{\mathrm{x}-\varepsilon_{1}(\eta) \mathrm{p}}{\varepsilon_{1}(\eta) \varepsilon_{2}(\eta)}\right)\right)=U_{0,1}(\mathrm{x}) \quad \forall \mathrm{x} \in \bar{\Omega} \backslash\{0\} .
$$

Now (3.14), 3.15), and (3.16) imply that $u_{0,1}^{\#}(\mathrm{x})=U_{0,1}(\mathrm{x})$ for all $\mathrm{x} \in \bar{\Omega} \backslash\{0\}$. Since both $u_{0,1}^{\#}$ and $U_{0,1}$ are continuous functions on $\bar{\Omega}$, it follows that

$$
u_{0,1}^{\#}=U_{0,1} \text { on the whole of } \bar{\Omega} .
$$

Then by 1.7 we compute that

$$
\begin{aligned}
u_{\boldsymbol{\varepsilon}(\eta)}(\mathrm{x})-W_{\boldsymbol{\varepsilon}(\eta),(0,1)}^{\#}(\mathrm{x})= & -v_{\mathbf{0}}\left(\frac{\varsigma(\mathrm{x})-\varepsilon_{1}(\eta) \mathrm{p}}{\varepsilon_{1}(\eta) \varepsilon_{2}(\eta)}\right)-\varepsilon_{2}(\eta) v_{0,1}^{\#}\left(\frac{\mathrm{x}-\varepsilon_{1}(\eta) \mathrm{p}}{\varepsilon_{1}(\eta) \varepsilon_{2}(\eta)}\right) \\
& +\sum_{(i, j)>(0,1)} \varepsilon_{1}^{i}(\eta) \varepsilon_{2}^{j}(\eta) U_{i, j}(\mathrm{x}) \\
& +\sum_{(i, j) \geq(0,1)} \varepsilon_{1}^{i}(\eta) \varepsilon_{2}^{j}(\eta) V_{i, j}\left(\frac{\mathrm{x}-\varepsilon_{1}(\eta) \mathrm{p}}{\varepsilon_{1}(\eta) \varepsilon_{2}(\eta)}\right) \\
& -\sum_{(i, j) \geq(0,1)} \varepsilon_{1}^{i}(\eta) \varepsilon_{2}^{j}(\eta) V_{i, j}\left(\frac{\varsigma(\mathrm{x})-\varepsilon_{1}(\eta) \mathrm{p}}{\varepsilon_{1}(\eta) \varepsilon_{2}(\eta)}\right) \quad \forall \mathrm{x} \in \overline{\Omega_{\varepsilon(\eta)}} .
\end{aligned}
$$

It follows that for $x=0$ we have

$$
u_{\boldsymbol{\varepsilon}(\eta)}(0)-W_{\boldsymbol{\varepsilon}(\eta),(0,1)}^{\#}(0)=-v_{\mathbf{0}}\left(-\frac{\mathrm{p}}{\varepsilon_{2}(\eta)}\right)+\varepsilon_{2}(\eta) v_{0,1}^{\#}\left(-\frac{\mathrm{p}}{\varepsilon_{2}(\eta)}\right)+o\left(\varepsilon_{2}(\eta)\right) \quad \text { as } \eta \rightarrow 0 .
$$

Then, by the membership of $v_{0,1}^{\#}$ in $\mathscr{C}_{0}\left(\mathbb{R}^{3} \backslash \omega\right)$ and by equality $v_{\mathbf{0}}(\mathrm{X})=1 /|\mathrm{X}|$, we have

$$
\lim _{\eta \rightarrow 0} \frac{1}{\varepsilon_{2}(\eta)}\left(u_{\varepsilon(\eta)}(0)-W_{\varepsilon(\eta),(0,1)}^{\#}(0)\right)=-\frac{1}{|\mathrm{p}|} \neq 0
$$

The latter limit relation contradicts (3.14) and the remark is proved.

To have $v_{\mathbf{0}}(\mathrm{X})=1 /|\mathrm{X}|$ as in Example 3.3 one may for instance consider the case where $\omega$ is the unit ball in $\mathbb{R}^{3}$ and $g^{\mathrm{i}}$ is identically equal to 1 and $g^{\mathrm{o}}=0$. However, in Example 3.3 the condition that $v_{\mathbf{0}}(\mathrm{X})=1 /|\mathrm{X}|$ can be relaxed and replaced by the assumption that

$$
\lim _{\mathbf{X} \rightarrow \infty}|\mathbf{X}| v_{\mathbf{0}}(\mathbf{X}) \neq 0
$$

One can show that $\lim _{\mathbf{X} \rightarrow \infty}|\mathbf{X}| v_{\mathbf{0}}(\mathbf{X})=0$ if and only if $v_{\mathbf{0}}$ is given by a double layer potential, that is

$$
\text { there is } \psi \in \mathscr{C}^{1, \alpha}(\partial \omega) \text { such that } v_{\mathbf{0}}=w_{S_{n}}^{e}[\partial \omega, \psi] \text {, }
$$


or, equivalently, if and only if

$$
\int_{\partial \omega} \mathbf{n}_{\omega} \cdot \nabla v_{\mathbf{0}} d \sigma=0
$$

As a consequence, the set of the boundary data $\left(g^{\mathrm{i}}, g^{\mathrm{o}}\right)$ for which (3.17) holds is generic in $\mathscr{C}^{1, \alpha}(\partial \omega) \times \mathscr{C}^{1, \alpha}(\partial \Omega)$, and, generically, we cannot expect to have approximations without reflected fast variable terms.

\section{The multi-scale asymptotic approach}

Taking $N=0$ in the approximation $(1.8)$ we have

$$
u_{\boldsymbol{\varepsilon}}(\mathrm{x})=u_{\mathbf{0}}(\mathrm{x})+v_{\mathbf{0}}\left(\frac{\mathrm{x}-\varepsilon_{1} \mathrm{p}}{\varepsilon_{1} \varepsilon_{2}}\right)-v_{\mathbf{0}}\left(\frac{\varsigma(\mathrm{x})-\varepsilon_{1} \mathrm{p}}{\varepsilon_{1} \varepsilon_{2}}\right)+O\left(\varepsilon_{1}+\varepsilon_{2}\right) \quad \text { as } \boldsymbol{\varepsilon} \rightarrow \mathbf{0},
$$

and the coefficients $u_{\mathbf{0}}$ and $v_{\mathbf{0}}$ can be computed solving the boundary value problems 1.2 and (1.4), respectively. Our aim in this section is to show how one can determine the next coefficients of (1.8). Namely, the $U_{i, j}$ 's and $V_{i, j}$ 's with $i+j>0$.

One way to do it would be by exploiting the boundary integral form of problem (1.1) (cf. [1]) to obtain an explicit expansion of the real analytic map $\mathrm{M} \equiv\left(\mathrm{M}_{1}, \mathrm{M}_{2}\right)$ and then pass from integral representation in Lemma 3.1 to deduce the expressions for the $U_{i, j}$ 's and $V_{i, j}$ 's. Similar computations have been presented in [6], 3], [4], and [5] in the context of other perturbation problems.

In the present paper we opt for a different and probably more direct approach: we follow the ideas of the multi-scale asymptotic expansions method of [2] and we aim at deriving asymptotic approximations of the solution combining macroscopic scale expansions (that is, away from the origin of $\mathbb{R}^{n}$ ) and microscopic scale expansions (close to the origin of $\mathbb{R}^{n}$ ). Typically, this idea is realized through an iterative process where one corrects a misfit on the outer boundary by means of a macroscopic term that will produce an error on the inclusion, then one takes care of the new error on the inclusion adding a fast variable term that in turn produces a new error on the outer boundary, and so on. If propertly formulated, the process will produce a better approximation at every iterative step. In our specific problem the process is complicated by the presence of the reflected fast variable terms (we have observed in Example 3.3 that such terms cannot in general be omitted). In particular, every time that we introduce a corrector in the fast variable, we have to add also its reflected counterpart. Both terms will produce a misfit on the outer boundary, but in addition, the reflected fast variable term will produce a new error on the inclusion. The resulting algorithm is described in Subsection 4.2 here below, where we also present some explicit computations in the case of dimension $n=3$. Before that, we introduce in the following Subsection 4.1 some series expansions that will be later exploited and we introduce the expansion operators $E_{\Omega}$ and $E_{\omega}$.

\subsection{Preliminary computations and notation}

\subsubsection{Some useful expansions}

Expansions on $\partial \omega_{\varepsilon}$ for functions in $\mathscr{C}_{\mathrm{h}}^{1, \alpha}(\bar{\Omega})$. If $u \in \mathscr{C}_{\mathrm{h}}^{1, \alpha}(\bar{\Omega})$ has an analytic extension in the neighborhood of 0 then the map which takes $\left(\varrho_{1}, \varrho_{2}\right)$ to the function

$$
\partial \omega \ni X \mapsto u\left(\varrho_{1} \mathrm{p}+\varrho_{2} X\right)
$$


is real analytic from a neighborhood of 0 to $\mathscr{C}^{1, \alpha}(\partial \omega)$ (cf. Valent [15, Thm. 5.2, p. 44]). Accordingly, there are coefficients $\mathfrak{u}_{i, j}^{\omega} \in \mathscr{C}^{1, \alpha}(\partial \omega)$ such that

$$
u\left(\varrho_{1} \mathrm{p}+\varrho_{2} X\right)=\sum_{i, j \geq 0} \varrho_{1}^{i} \varrho_{2}^{j} \mathfrak{u}_{i, j}^{\omega}(X)
$$

where the series converges in $\mathscr{C}^{1, \alpha}(\partial \omega)$ for $\varrho_{1}$ and $\varrho_{2}$ close to 0 (here and in what follows we omit to indicate the dependence of the expansions' coefficients on the vector $p$, that is considered as fixed). Then, replacing $\varrho_{1}$ with $\varepsilon_{1}$ and $\varrho_{2}$ with $\varepsilon_{1} \varepsilon_{2}$, we have

$$
u\left(\varepsilon_{1} \mathrm{p}+\varepsilon_{1} \varepsilon_{2} \mathrm{X}\right)=\sum_{i, j \geq 0} \varepsilon_{1}^{i}\left(\varepsilon_{1} \varepsilon_{2}\right)^{j} \mathfrak{u}_{i, j}^{\omega}(\mathbf{X})
$$

and, rearranging the sum, we obtain

$$
u\left(\varepsilon_{1} \mathrm{p}+\varepsilon_{1} \varepsilon_{2} \mathrm{X}\right)=\sum_{i \geq j \geq 0} \varepsilon_{1}^{i} \varepsilon_{2}^{j}[u]_{i, j}^{\omega}(\mathrm{X}) .
$$

for certain coefficients $[u]_{i, j}^{\omega} \in \mathscr{C}^{1, \alpha}(\partial \omega)$. The first ones are:

$$
\begin{aligned}
& {[u]_{0,0}^{\omega}(\mathrm{X})=u(0), \quad[u]_{1,0}^{\omega}(\mathrm{X})=\nabla u(0) \cdot \mathrm{p}, \quad[u]_{1,1}^{\omega}(\mathrm{X})=\nabla u(0) \cdot \mathbf{X},} \\
& {[u]_{2,0}^{\omega}(\mathrm{X})=\frac{1}{2} D^{2} u(0) \cdot(\mathrm{p}, \mathrm{p}), \quad[u]_{2,1}^{\omega}(\mathrm{X})=D^{2} u(0) \cdot(\mathrm{p}, \mathrm{X}), \quad[u]_{2,2}^{\omega}(\mathrm{X})=\frac{1}{2} D^{2} u(0) \cdot(\mathrm{X}, \mathrm{X}) .}
\end{aligned}
$$

Expansions on $\partial_{+} \Omega$ for rescaled functions in $\mathscr{C}_{\mathrm{h}}^{1, \alpha}\left(\mathbb{R}^{n} \backslash \omega\right)$. Now let $v \in \mathscr{C}_{\mathrm{h}}^{1, \alpha}\left(\mathbb{R}^{n} \backslash \omega\right)$ and let $v^{*}$ denote the Kelvin transform of $v$, so that

$$
v(\mathrm{X})=|\mathrm{X}|^{2-n} v^{*}\left(\frac{\mathrm{X}}{|\mathrm{X}|^{2}}\right) \quad \forall \mathrm{X} \in \mathbb{R}^{n} \backslash \omega .
$$

Since $v$ is harmonic at infinity, $v^{*}$ has an harmonic (and hence analytic) extension in a neighborhood of 0 , which we still denote by $v^{*}$ (cf. Folland [7, p. 114]). Moreover, for $x \in \overline{\partial_{+} \Omega}$, we have

$$
v\left(\frac{\mathrm{x}-\varepsilon_{1} \mathrm{p}}{\varepsilon_{1} \varepsilon_{2}}\right)=\left|\varepsilon_{1} \varepsilon_{2}\right|^{n-2}\left|\mathrm{x}-\varepsilon_{1} \mathrm{p}\right|^{2-n} v^{*}\left(\varepsilon_{1} \varepsilon_{2} \frac{\mathrm{x}-\varepsilon_{1} \mathrm{p}}{\left|\mathrm{x}-\varepsilon_{1} \mathrm{p}\right|^{2}}\right) .
$$

Thanks to the fact that $x \in \overline{\partial_{+} \Omega}$ stays far away from 0 , one can show that the map which takes $\left(\varrho_{1}, \varrho_{2}\right)$ to the function

$$
\overline{\partial_{+} \Omega} \ni \mathrm{x} \mapsto\left|\mathrm{x}-\varrho_{1} \mathrm{p}\right|^{2-n} v^{*}\left(\varrho_{2} \frac{\mathrm{x}-\varrho_{1} \mathrm{p}}{\left|\mathrm{x}-\varrho_{1} \mathrm{p}\right|^{2}}\right)
$$

is real analytic from a neighborhood of $\mathbf{0}$ in $\mathbb{R}^{2}$ to $\mathscr{C}^{1, \alpha}\left(\overline{\partial_{+} \Omega}\right)$ (cf. Valent [15, Thm. 5.2, p. 44]). As a consequence, there are coefficients $\mathfrak{v}_{i, j}^{\Omega} \in \mathscr{C}^{1, \alpha}\left(\overline{\partial_{+} \Omega}\right)$ such that

$$
v\left(\frac{\mathrm{x}-\varrho_{1} \mathrm{p}}{\varrho_{2}}\right)=\varrho_{2}^{n-2} \sum_{i, j \geq 0} \varrho_{1}^{i} \varrho_{2}^{j} \mathfrak{v}_{i, j}^{\Omega}(\mathrm{x}),
$$


for $\left(\varrho_{1}, \varrho_{2}\right)>\mathbf{0}$ small enough. Here the series converges in $\mathscr{C}^{1, \alpha}\left(\overline{\partial_{+} \Omega}\right)$ for $\left(\varrho_{1}, \varrho_{2}\right)$ in a neighborhood of $\mathbf{0}$. Then, replacing $\varrho_{1}$ with $\varepsilon_{1}$ and $\varrho_{2}$ with $\varepsilon_{1} \varepsilon_{2}$ and rearranging the sum, we find that there are coefficients $[v]_{i, j}^{\Omega} \in \mathscr{C}^{1, \alpha}\left(\overline{\partial_{+} \Omega}\right)$ such that

$$
v\left(\frac{\mathrm{x}-\varepsilon_{1} \mathrm{p}}{\varepsilon_{1} \varepsilon_{2}}\right)=\sum_{i \geq j \geq n-2} \varepsilon_{1}^{i} \varepsilon_{2}^{j}[v]_{i, j}^{\Omega}(\mathrm{x}),
$$

for $\varepsilon>\mathbf{0}$ small enough. Again, the series converges in $\mathscr{C}^{1, \alpha}\left(\overline{\partial_{+} \Omega}\right)$ for $\varepsilon$ in a suitable neighborhood of $\mathbf{0}$. A similar argument holds with $x \in \overline{\varsigma\left(\partial_{+} \Omega\right)}$ instead of $\overline{\partial_{+} \Omega}$, the key point of the computation being that $x$ stays in a compact manifold which does not contain 0 . As a consequence, we can find coefficients $[v]_{i, j}^{\varsigma(\Omega)} \in \mathscr{C}^{1, \alpha}\left(\overline{\varsigma\left(\partial_{+} \Omega\right)}\right)$ such that

$$
v\left(\frac{\varsigma(\mathrm{x})-\varepsilon_{1} \mathrm{p}}{\varepsilon_{1} \varepsilon_{2}}\right)=\sum_{i \geq j \geq n-2} \varepsilon_{1}^{i} \varepsilon_{2}^{j}[v]_{i, j}^{\varsigma(\Omega)}(\varsigma(\mathrm{x})),
$$

for $\varepsilon>\mathbf{0}$ small enough. One can also verify that the extension by 0 of the map

$$
\overline{\partial_{+} \Omega} \ni \mathbf{x} \mapsto[v]_{i, j}^{\Omega}(\mathrm{x})-[v]_{i, j}^{\varsigma(\Omega)}(\varsigma(\mathrm{x}))
$$

belongs to $\mathscr{C}^{1, \alpha}(\partial \Omega)$ for all $i \geq j \geq n-2$.

Finally, we observe that a function $v \in \mathscr{C}_{\mathrm{h}}^{1, \alpha}\left(\mathbb{R}^{n} \backslash \omega\right)$ can be written as the sum of homogeneous harmonic functions $\underline{v}_{k}$ of degree $-k$, for $k \geq n-2$. Namely, we have

$$
v(\mathbf{X})=\sum_{k \geq n-2} \underline{v}_{k}(\mathbf{X}) \quad \forall \mathbf{X} \in \mathbb{R}^{n} \backslash \omega .
$$

(This can be verified by introducing a sphere $\partial \mathcal{B}(0, R)$ in $\mathbb{R}^{n} \backslash \omega$ and by considering the decomposition of $u_{\mid \partial \mathcal{B}(0, R)}$ into spherical harmonics.) Then, we have

$$
v\left(\frac{\mathrm{x}-\varepsilon_{1} \mathrm{p}}{\varepsilon_{1} \varepsilon_{2}}\right)=\sum_{k \geq n-2}\left(\varepsilon_{1} \varepsilon_{2}\right)^{k} \underline{v}_{k}\left(\mathrm{x}-\varepsilon_{1} \mathrm{p}\right) \quad \forall \mathrm{x} \in \overline{\partial_{+} \Omega} .
$$

Now, for each $k \geq n-2$ the map from a neighborhood of 0 in $\mathbb{R}$ to $\mathscr{C}^{1, \alpha}\left(\overline{\partial_{+} \Omega}\right)$ which takes $\varepsilon_{1}$ to the function $\underline{v}_{k}\left(\cdot-\varepsilon_{1} \mathrm{p}\right)$ is real analytic. Accordingly, there is a sequence of coefficients $\left[\underline{v}_{k}\right]_{h}^{\Omega} \in \mathscr{C}^{1, \alpha}\left(\overline{\partial_{+} \Omega}\right)$ such that

$$
\underline{v}_{k}\left(\mathrm{x}-\varepsilon_{1} \mathrm{p}\right)=\sum_{h \geq 0} \varepsilon_{1}^{h}\left[\underline{v}_{k}\right]_{h}^{\Omega}(\mathrm{x}) .
$$

Then, comparing 4.5 with 4.8 we can express the coefficients $[v]_{i, j}^{\Omega}$ in terms of expansions terms of the homogeneous harmonic functions $\underline{v}_{k}$. For example, for the first coefficient of 4.5 we have

$$
[v]_{n-2, n-2}^{\Omega}(\mathrm{x})=\underline{v}_{n-2}(\mathrm{x}) \quad \forall \mathrm{x} \in \overline{\partial_{+} \Omega} .
$$

A similar computation can be performed also for the coefficients of (4.6). The first one will be

$$
[v]_{n-2, n-2}^{\varsigma(\Omega)}(\mathrm{x})=\underline{v}_{n-2}(\varsigma(\mathrm{x})) \quad \forall \mathrm{x} \in \overline{\partial_{+} \Omega} .
$$


Expansions on $\partial \omega_{\varepsilon}$ for reflected rescaled functions in $\mathscr{C}_{\mathrm{h}}^{1, \alpha}\left(\mathbb{R}^{n} \backslash \omega\right)$. In what follows, we will also need to consider the expansion on $\partial \omega_{\varepsilon}$ of functions which are harmonic in $\mathbb{R}^{n} \backslash \varsigma\left(\omega_{\varepsilon}\right)$. Namely, for $v \in \mathscr{C}_{\mathrm{h}}^{1, \alpha}\left(\mathbb{R}^{n} \backslash \omega\right)$, we consider the expansion of

$$
v\left(\frac{\varsigma\left(\varepsilon_{1} \mathrm{p}+\varepsilon_{1} \varepsilon_{2} \mathrm{X}\right)-\varepsilon_{1} \mathrm{p}}{\varepsilon_{1} \varepsilon_{2}}\right) .
$$

We observe that

$$
\frac{\varsigma\left(\varepsilon_{1} \mathrm{p}+\varepsilon_{1} \varepsilon_{2} \mathrm{X}\right)-\varepsilon_{1} \mathrm{p}}{\varepsilon_{1} \varepsilon_{2}}=\frac{1}{\varepsilon_{2}}\left(\varsigma(\mathrm{p})-\mathrm{p}+\varepsilon_{2} \varsigma(\mathrm{X})\right) .
$$

As a consequence, the argument of 4.9 depends only on $\varepsilon_{2}$ and not on $\varepsilon_{1}$. Moreover, by (4.4) and 4.10) we deduce that

$$
v\left(\frac{\varsigma\left(\varepsilon_{1} \mathrm{p}+\varepsilon_{1} \varepsilon_{2} \mathrm{X}\right)-\varepsilon_{1} \mathrm{p}}{\varepsilon_{1} \varepsilon_{2}}\right)=\left|\varepsilon_{2}\right|^{n-2}\left(\varsigma(\mathrm{p})-\mathrm{p}+\varepsilon_{2} \varsigma(\mathrm{X})\right)^{2-n} v^{*}\left(\varepsilon_{2} \frac{\varsigma(\mathrm{p})-\mathrm{p}+\varepsilon_{2} \varsigma(\mathrm{X})}{\left|\varsigma(\mathrm{p})-\mathrm{p}+\varepsilon_{2} \varsigma(\mathrm{X})\right|^{2}}\right) .
$$

Since $v^{*}$ is analytic in a neighborhood of 0 and $\varsigma(\mathrm{p})-\mathrm{p}=-2 p_{n} \mathrm{e}_{n} \neq 0$, one verifies that the map which takes $\varepsilon_{2}$ to the function

$$
\partial \omega \ni \mathbf{X} \mapsto\left(\varsigma(\mathrm{p})-\mathrm{p}+\varepsilon_{2} \varsigma(\mathrm{X})\right)^{2-n} v^{*}\left(\varepsilon_{2} \frac{\varsigma(\mathrm{p})-\mathrm{p}+\varepsilon_{2} \varsigma(\mathrm{X})}{\left|\varsigma(\mathrm{p})-\mathrm{p}+\varepsilon_{2} \varsigma(\mathrm{X})\right|^{2}}\right)
$$

is real analytic from a neighborhood of 0 in $\mathbb{R}$ to $\mathscr{C}^{1, \alpha}(\partial \omega)$. We deduce that there are coefficients $[v]_{j}^{\omega, \varsigma(\omega)} \in \mathscr{C}^{1, \alpha}(\partial \omega)$ such that

$$
v\left(\frac{\varsigma\left(\varepsilon_{1} \mathrm{p}+\varepsilon_{1} \varepsilon_{2} \mathrm{X}\right)-\varepsilon_{1} \mathrm{p}}{\varepsilon_{1} \varepsilon_{2}}\right)=\sum_{j \geq n-2} \varepsilon_{2}^{j}[v]_{j}^{\omega, \varsigma(\omega)}(\mathrm{X}),
$$

for $\varepsilon>\mathbf{0}$ small enough. As for the $[v]_{i, j}^{\Omega}$ 's and $[v]_{i, j}^{\varsigma(\Omega)}$ 's, also for the coefficients $[v]_{j}^{\omega, \varsigma(\omega)}$ we can find expressions in terms of the homogeneous functions $\underline{v}_{k}$. Indeed, by 4.7) and 4.10) we have

$$
v\left(\frac{\varsigma\left(\varepsilon_{1} \mathrm{p}+\varepsilon_{1} \varepsilon_{2} \mathrm{X}\right)-\varepsilon_{1} \mathrm{p}}{\varepsilon_{1} \varepsilon_{2}}\right)=\sum_{k \geq n-2} \varepsilon_{2}^{k} \underline{v}_{k}\left(\varsigma(\mathrm{p})-\mathrm{p}+\varepsilon_{2} \varsigma(\mathrm{X})\right) .
$$

Since $\varsigma(\mathrm{p})-\mathrm{p} \neq 0$ and the $\underline{v}_{k}$ 's are analytic away from 0 , we can take their Taylor series at $\varsigma(\mathrm{p})-\mathrm{p}$ and then compare 4.11 with 4.12 to obtain expressions for the $[v]_{j}^{\omega, \varsigma(\omega)}$, s. The first one is

$$
[v]_{n-2}^{\omega, \varsigma(\omega)}(\mathbf{X})=\underline{v}_{n-2}(\varsigma(\mathrm{p})-\mathrm{p}), \quad \forall \mathbf{X} \in \partial \omega
$$

\subsubsection{Extension operators}

We will exploit the extension operators $E_{\Omega}$ and $E_{\omega}$ defined as follows: $E_{\Omega}$ takes a function $\phi \in \mathscr{C}^{1, \alpha}(\partial \Omega)$ to the unique solution $u \in \mathscr{C}_{\mathrm{h}}^{1, \alpha}(\bar{\Omega})$ of the Dirichlet boundary value problem

$$
\begin{cases}\Delta u=0 & \text { in } \Omega, \\ u=\phi & \text { on } \partial \Omega,\end{cases}
$$


and $E_{\omega}$ takes a function $\psi \in \mathscr{C}^{1, \alpha}(\partial \omega)$ to the unique solution $v \in \mathscr{C}_{\mathrm{h}}^{1, \alpha}\left(\mathbb{R}^{n} \backslash \omega\right)$ of the exterior Dirichlet boundary value problem

$$
\begin{cases}\Delta v=0 & \text { in } \mathbb{R}^{n} \backslash \bar{\omega} \\ v=\psi & \text { on } \partial \omega \\ \lim _{\mathbf{X} \rightarrow \infty} v(\mathbf{X})=0 . & \end{cases}
$$

\subsection{The iterative procedure}

We now describe an algorithm that produces a better approximation at every iteration. To do so, we have to decide what we intend by 'better approximation'. Indeed, when one has only one perturbation parameter this is immediately evident: an error of order $\varepsilon^{3}$ is better than an error of order $\varepsilon$. With two parameters $\varepsilon_{1}$ and $\varepsilon_{2}$ the question is more complicated. If we let the pair $\left(\varepsilon_{1}, \varepsilon_{2}\right)$ tend to $(0,0)$ along a curve in $\mathbb{R}^{2}$, then a combination of $\varepsilon_{1}$ and $\varepsilon_{2}$ may or may not be smaller than another depending on the specific curve. For example, if we take both $\varepsilon_{1}=\varepsilon$ and $\varepsilon_{2}=\varepsilon$, then an error of order $\varepsilon_{1}^{3} \varepsilon_{2}$ would be better than an error of order $\varepsilon_{1} \varepsilon_{2}^{2}$, if instead $\varepsilon_{1}=\varepsilon$ and $\varepsilon_{2}=\varepsilon^{2}$, then it is the second error to be preferable. It seems that there is not a unique canonical way to solve the problem. Then we have to introduce a suitable convention. In this paper, we decide that an error of order $\varepsilon_{1}^{i} \varepsilon_{2}^{j}$ is preferable to one of order $\varepsilon_{1}^{k} \varepsilon_{2}^{h}$ if $i+j>h+k$. In other words, we say that

$$
\text { an error in } O\left(\sum_{i+j=M} \varepsilon_{1}^{i} \varepsilon_{2}^{j}\right) \text { is smaller than an error in } O\left(\sum_{i+j=N} \varepsilon_{1}^{i} \varepsilon_{2}^{j}\right) \text { if } M>N \text {. }
$$

An advantage of condition 4.15 is that the membership of an error in $O\left(\sum_{i+j=N} \varepsilon_{1}^{i} \varepsilon_{2}^{j}\right)$ is independent on the possible parametrization of $\left(\varepsilon_{1}, \varepsilon_{2}\right)$ along a curve. However, for the purpose of determining the coefficients $U_{i, j}$ and $V_{i, j}$ in the expansion, criteria 4.15) is as good as any other one could take.

The algorithm. Suppose that we have already identified the coefficients of an approximation with a remainder in $O\left(\sum_{i+j=N} \varepsilon_{1}^{i} \varepsilon_{2}^{j}\right)$. To determine the next coefficients we proceed as follows:

Step 1. We consider the approximation that we have and we use the expansions 4.2 , (4.5), (4.6), and 4.11) to determine the principal parts of the misfits on $\partial_{+} \Omega$ and $\partial \omega_{\varepsilon}$. We note that our procedure never gives errors on $\partial_{0} \Omega$. In other words, at any step all the approximations will be exact on $\partial_{0} \Omega$. Then, there might be two different situations:

(a) The errors on $\partial_{+} \Omega$ and $\partial \omega_{\varepsilon}$ are equivalent. That is, they are both in $O\left(\sum_{i+j=M} \varepsilon_{1}^{i} \varepsilon_{2}^{j}\right)$ for some $M \geq N$ and neither one is in $O\left(\sum_{i+j=M+1} \varepsilon_{1}^{i} \varepsilon_{2}^{j}\right)$;

(b) The error on $\partial \omega_{\varepsilon}$ is bigger than the error on $\partial_{+} \Omega$. The error on $\partial \omega_{\varepsilon}$ can be generated by the slow variable terms, as it happens in the classical applications of the multi-scale expansion method, but also by the reflected fast variable terms.

In principle one may expect another case, that is, the case where the main error is on $\partial_{+} \Omega$. However, the application of the present algorithm never generates such a situation (as we shall also see in the iterations below). 
Step 2. Depending if we have case (a) or (b) in Step 1, we proceed with the following Step 2.(a) or Step 2.(b):

(a) If we have reached the desired precision, we stop here the iterative procedure, otherwise we correct the main error on $\partial_{+} \Omega$. To do so, first we extend the misfit on $\partial_{+} \Omega$ by 0 on the whole of $\partial \Omega$ and then we exploit $E_{\Omega}$ to construct a corrector. Adding such corrector to the approximation that we already have generates a new error on $\partial \omega_{\varepsilon}$;

(b) We correct the principal part of the error on $\partial \omega_{\varepsilon}$. We construct correctors extending the traces on $\partial \omega_{\varepsilon}$ by means of $E_{\omega}$. In view of Remark 3.3 , for any new fast variable term that we add to our approximation we must also add a corresponding reflected fast variable term. This operation generates new errors on $\partial_{+} \Omega$ and $\partial \omega_{\varepsilon}$, but not on $\partial_{0} \Omega$ because the contribution of the fast variable term and of the reflected fast variable term cancel one another on that part of the outer boundary.

When Step 2 is completed we go back to Step 1 and start over again, or, if we were in case (a), we can decide to stop the iterative procedure. If we stop with an approximation that has remainder in $O\left(\sum_{i+j=M} \varepsilon_{1}^{i} \varepsilon_{2}^{j}\right)$, then by Proposition 3.2 the coefficients of such approximation must coincide with the coefficients $U_{i, j}$ and $V_{i, j}$ with $\imath+j \leq M-1$. As a consequence, we will be able to identify all the coefficients $U_{i, j}$ and $V_{i, j}$ with $i+j \leq M-1$ as the first terms of the Taylor's expansion of solutions of certain boundary value problems.

We now present an explicit computation. For the sake of simplicity in the exposition, we confine ourselves to the 3 -dimensional case $(n=3)$ and we stop the iteration with a remainder of order 3 , that is, in $O\left(\sum_{i+j=3} \varepsilon_{1}^{i} \varepsilon_{2}^{j}\right)$. Other dimensions can be studied and further approximations can be obtained by a straightforward modification of what we do here below.

\section{First iteration}

Step 1. In view of 4.1), we start with the approximation

$$
u_{0,0}(\mathrm{x})+v_{0,0}\left(\frac{\mathrm{x}-\varepsilon_{1} \mathrm{p}}{\varepsilon_{1} \varepsilon_{2}}\right)-v_{0,0}\left(\frac{\varsigma(\mathrm{x})-\varepsilon_{1} \mathrm{p}}{\varepsilon_{1} \varepsilon_{2}}\right) \quad \forall \mathrm{x} \in \overline{\Omega_{\varepsilon}},
$$

where $u_{0,0}=u_{\mathbf{0}}$ is the unique solution of 1.2 and $v_{0,0}=v_{\mathbf{0}}-g^{o}(0)$, with $v_{\mathbf{0}}$ as in 1.4 (we modify $v_{\mathbf{0}}$ to have a function $v_{0,0}$ that is harmonic at infinity). Since $u_{0,0}(\mathrm{x})=g^{o}(\mathrm{x})$ for $x \in \partial_{+} \Omega$, the trace of 4.16 on $\partial_{+} \Omega$ is given by

$$
g^{o}(\mathrm{x})+v_{0,0}\left(\frac{\mathrm{x}-\varepsilon_{1} \mathrm{p}}{\varepsilon_{1} \varepsilon_{2}}\right)-v_{0,0}\left(\frac{\varsigma(\mathrm{x})-\varepsilon_{1} \mathrm{p}}{\varepsilon_{1} \varepsilon_{2}}\right) \quad \forall \mathrm{x} \in \partial_{+} \Omega .
$$

Then we have on $\partial_{+} \Omega$ a misfit

$$
v_{0,0}\left(\frac{\mathrm{x}-\varepsilon_{1} \mathrm{p}}{\varepsilon_{1} \varepsilon_{2}}\right)-v_{0,0}\left(\frac{\varsigma(\mathrm{x})-\varepsilon_{1} \mathrm{p}}{\varepsilon_{1} \varepsilon_{2}}\right) \quad \forall \mathrm{x} \in \partial_{+} \Omega .
$$

Using the expansions 4.5 and 4.6 one verifies that the principal part of the error on $\partial_{+} \Omega$ is given by

$$
\varepsilon_{1} \varepsilon_{2}\left[v_{0,0}\right]_{1,1}^{\Omega}(\mathrm{x})-\varepsilon_{1} \varepsilon_{2}\left[v_{0,0}\right]_{1,1}^{\zeta(\Omega)}(\varsigma(\mathrm{x})) \quad \forall \mathrm{x} \in \partial_{+} \Omega .
$$


We now pass to consider the error on $\partial \omega_{\varepsilon}$. Since $v_{0}=v_{0,0}+g^{o}(0)$ solves the boundary value problem (1.4), the trace of (4.16) on $\partial \omega_{\varepsilon}$ is given by

$$
u_{0,0}\left(\varepsilon_{1} \mathbf{p}+\varepsilon_{1} \varepsilon_{2} \mathbf{X}\right)+g^{i}(\mathbf{X})-g^{o}(0)+v_{0,0}\left(\frac{\varsigma\left(\varepsilon_{1} \mathbf{p}+\varepsilon_{1} \varepsilon_{2} \mathbf{X}\right)-\varepsilon_{1} \mathbf{p}}{\varepsilon_{1} \varepsilon_{2}}\right) \quad \forall \mathbf{X} \in \partial \omega .
$$

Accordingly, we have on $\partial \omega_{\varepsilon}$ a misfit

$$
u_{0,0}\left(\varepsilon_{1} \mathrm{p}+\varepsilon_{1} \varepsilon_{2} \mathrm{X}\right)-g^{o}(0)+v_{0,0}\left(\frac{\varsigma\left(\varepsilon_{1} \mathrm{p}+\varepsilon_{1} \varepsilon_{2} \mathrm{X}\right)-\varepsilon_{1} \mathrm{p}}{\varepsilon_{1} \varepsilon_{2}}\right) \quad \forall \mathbf{X} \in \partial \omega
$$

By the expansion 4.2 the main contribution of $u_{0,0}\left(\varepsilon_{1} \mathrm{p}+\varepsilon_{1} \varepsilon_{2} \mathrm{X}\right)$ for $\varepsilon_{1}$ and $\varepsilon_{2}$ small is given by $\left[u_{0,0}\right]_{0,0}^{\omega}(\mathrm{X})=u_{0,0}(0)=g^{o}(0)$ (see also $4.3 \mathrm{n}$ ) and thus it is corrected by the term $-g^{o}(0)$ produced by $v_{0,0}(\mathrm{X})$. Then, one can exploit the expansions (4.2) and (4.11) to show that the principal part of the error that remains on $\partial \omega_{\varepsilon}$ is given by

$$
\varepsilon_{1}\left[u_{0,0}\right]_{1,0}^{\omega}(\mathbf{X})-\varepsilon_{2}\left[v_{0,0}\right]_{1}^{\omega, \varsigma(\omega)}(\mathbf{X}) \quad \forall \mathbf{X} \in \partial \omega .
$$

As one can see, the error on $\partial \omega_{\varepsilon}$ is bigger than the one on $\partial_{+} \Omega$ computed in (4.17): we are in case (b).

Step 2.(b). We proceed to correct the misfits on $\partial \omega_{\varepsilon}$. To do so, we introduce the functions

$$
v_{1,0}(\mathbf{X})=-E_{\omega}\left(\left[u_{0,0}\right]_{1,0}^{\omega}\right)(\mathbf{X}) \quad \text { and } \quad v_{0,1}(\mathbf{X})=E_{\omega}\left(\left[v_{0,0}\right]_{1}^{\omega, \varsigma(\omega)}\right)(\mathbf{X}) \quad \forall \mathbf{X} \in \mathbb{R}^{3} \backslash \omega .
$$

With these our next approximation is

$$
\begin{aligned}
u_{0,0}(\mathrm{x})+v_{0,0}\left(\frac{\mathrm{x}-\varepsilon_{1} \mathrm{p}}{\varepsilon_{1} \varepsilon_{2}}\right)-v_{0,0} & \left(\frac{\varsigma(\mathrm{x})-\varepsilon_{1} \mathrm{p}}{\varepsilon_{1} \varepsilon_{2}}\right)+\varepsilon_{1} v_{1,0}\left(\frac{\mathrm{x}-\varepsilon_{1} \mathrm{p}}{\varepsilon_{1} \varepsilon_{2}}\right)-\varepsilon_{1} v_{1,0}\left(\frac{\varsigma(\mathrm{x})-\varepsilon_{1} \mathrm{p}}{\varepsilon_{1} \varepsilon_{2}}\right) \\
+ & \varepsilon_{2} v_{0,1}\left(\frac{\mathrm{x}-\varepsilon_{1} \mathrm{p}}{\varepsilon_{1} \varepsilon_{2}}\right)-\varepsilon_{2} v_{0,1}\left(\frac{\varsigma(\mathrm{x})-\varepsilon_{1} \mathrm{p}}{\varepsilon_{1} \varepsilon_{2}}\right) \quad \forall \mathrm{x} \in \overline{\Omega_{\varepsilon}} .
\end{aligned}
$$

\section{Second iteration}

Step 1. With the approximation (4.19) the main error on $\partial_{+} \Omega$ remains (4.17). Indeed, one can exploit the expansions (4.5) and (4.6) to verify that the misfit produced on $\partial_{+} \Omega$ by the new correctors are in $O\left(\sum_{i+j=3} \varepsilon_{1}^{i} \varepsilon_{2}^{j}\right)$ and thus they are smaller than 4.17). Then, we consider the trace of (4.19) on $\partial \omega_{\varepsilon}$ and exploit (4.2) and 4.11) to compute the principal part of the misfit (the computation is similar to the one described in Step 1 of the first iteration). We obtain

$$
\varepsilon_{1}^{2}\left[u_{0,0}\right]_{2,0}^{\omega}(\mathbf{X})+\varepsilon_{1} \varepsilon_{2}\left[u_{0,0}\right]_{1,1}^{\omega}(\mathbf{X})-\varepsilon_{2}^{2}\left[v_{0,0}\right]_{2}^{\omega, \varsigma(\omega)}(\mathbf{X})-\varepsilon_{1} \varepsilon_{2}\left[v_{1,0}\right]_{1}^{\omega, \varsigma(\omega)}(\mathbf{X})-\varepsilon_{2}^{2}\left[v_{0,1}\right]_{1}^{\omega, \varsigma(\omega)}(\mathbf{X})
$$

with $X \in \partial \omega$. As one can see, the errors on $\partial_{+} \Omega$ and $\partial \omega_{\varepsilon}$ are now equivalent. So, we are in case (a). 
Step 2.(a). We can decide to stop here the iteration and content ourselves with a remainder in $O\left(\sum_{i+j=2} \varepsilon_{1}^{i} \varepsilon_{2}^{j}\right)$, or we can proceed to correct the misfit 4.17) on $\partial_{+} \Omega$. We opt for the second choice and we introduce the corrector

$$
u_{1,1}(\mathrm{x})=-E_{\Omega}\left(\left[v_{0,0}\right]_{1,1}^{\Omega}(\cdot)-\left[v_{0,0}\right]_{1,1}^{\varsigma(\Omega)}(\varsigma(\cdot))\right)(\mathbf{x})
$$

where we understand that $\left[v_{0,0}\right]_{1,1}^{\Omega}(\cdot)-\left[v_{0,0}\right]_{1,1}^{\varsigma(\Omega)}(\varsigma(\cdot))$ is extended by 0 on $\partial_{0} \Omega$. With this, our next approximation is

$$
\begin{aligned}
u_{0,0}(\mathrm{x}) & +\varepsilon_{1} \varepsilon_{2} u_{1,1}(\mathrm{x})+v_{0,0}\left(\frac{\mathrm{x}-\varepsilon_{1} \mathrm{p}}{\varepsilon_{1} \varepsilon_{2}}\right)-v_{0,0}\left(\frac{\varsigma(\mathrm{x})-\varepsilon_{1} \mathrm{p}}{\varepsilon_{1} \varepsilon_{2}}\right)+\varepsilon_{1} v_{1,0}\left(\frac{\mathrm{x}-\varepsilon_{1} \mathrm{p}}{\varepsilon_{1} \varepsilon_{2}}\right) \\
& -\varepsilon_{1} v_{1,0}\left(\frac{\varsigma(\mathrm{x})-\varepsilon_{1} \mathrm{p}}{\varepsilon_{1} \varepsilon_{2}}\right)+\varepsilon_{2} v_{0,1}\left(\frac{\mathrm{x}-\varepsilon_{1} \mathrm{p}}{\varepsilon_{1} \varepsilon_{2}}\right)-\varepsilon_{2} v_{0,1}\left(\frac{\varsigma(\mathrm{x})-\varepsilon_{1} \mathrm{p}}{\varepsilon_{1} \varepsilon_{2}}\right) \quad \forall \mathrm{x} \in \overline{\Omega_{\varepsilon}} .
\end{aligned}
$$

\section{Third iteration}

Step 1. We now consider the trace of the approximation on $\partial_{+} \Omega$ and use the expansions (4.5) and 4.6 to compute the principal part of the misfit (see Step 1 of the first iteration). Doing so, we verify that the main part of the error of 4.22 on $\partial_{+} \Omega$ is given by

$$
\begin{aligned}
& \varepsilon_{1}^{2} \varepsilon_{2}\left[v_{0,0}\right]_{2,1}^{\Omega}(\mathrm{x})+\varepsilon_{1}^{2} \varepsilon_{2}\left[v_{0,0}\right]_{2,1}^{\varsigma(\Omega)}(\varsigma(\mathrm{x}))+\varepsilon_{1}^{2} \varepsilon_{2}\left[v_{1,0}\right]_{1,1}^{\Omega}(\mathrm{x}) \\
& -\varepsilon_{1}^{2} \varepsilon_{2}\left[v_{1,0}\right]_{1,1}^{\varsigma(\Omega)}(\varsigma(\mathrm{x}))+\varepsilon_{1} \varepsilon_{2}^{2}\left[v_{0,1}\right]_{1,1}^{\Omega}(\mathrm{x})-\varepsilon_{1} \varepsilon_{2}^{2}\left[v_{0,1}\right]_{1,1}^{\varsigma(\Omega)}(\varsigma(\mathrm{x})) \quad \forall \mathrm{x} \in \partial_{+} \Omega .
\end{aligned}
$$

Instead, the main part of the error on $\partial \omega_{\varepsilon}$ is still given by 4.20 . Indeed, $u_{1,1}(0)=0$ and thus $\left[u_{1,1}\right]_{0,0}^{\omega}=0$. In view of the expansion (4.2), it follows that the main contribution of $\varepsilon_{1} \varepsilon_{2} u_{1,1}$ on $\partial \omega_{\varepsilon}$ is $\varepsilon_{1}^{2} \varepsilon_{2}\left[u_{1,1}\right]_{1,0}^{\omega}$ which is smaller than $(4.20)$. Then, we observe that the misfit 4.20 on $\partial \omega_{\varepsilon}$ is bigger than the misfit 4.23 on $\partial_{+} \Omega$ : we deduce that we are in case (b).

Step 2.(b). Accordingly, the next correctors will be

$$
\begin{aligned}
& v_{2,0}(\mathbf{X})=-E_{\omega}\left(\left[u_{0,0}\right]_{2,0}^{\omega}\right)(\mathbf{X}), \\
& v_{1,1}(\mathbf{X})=-E_{\omega}\left(\left[u_{0,0}\right]_{1,1}^{\omega}-\left[v_{1,0}\right]_{1}^{\omega, \varsigma(\omega)}\right)(\mathbf{X}), \\
& v_{0,2}(\mathbf{X})=E_{\omega}\left(\left[v_{0,0}\right]_{2}^{\omega, \varsigma(\omega)}+\left[v_{0,1}\right]_{1}^{\omega, \varsigma(\omega)}\right)(\mathbf{X})
\end{aligned}
$$


for $X \in \mathbb{R}^{3} \backslash \omega$, which yield the approximation

$$
\begin{aligned}
u_{0,0}(\mathrm{x})+\varepsilon_{1} \varepsilon_{2} u_{1,1}(\mathrm{x}) & +v_{0,0}\left(\frac{\mathrm{x}-\varepsilon_{1} \mathrm{p}}{\varepsilon_{1} \varepsilon_{2}}\right)-v_{0,0}\left(\frac{\varsigma(\mathrm{x})-\varepsilon_{1} \mathrm{p}}{\varepsilon_{1} \varepsilon_{2}}\right) \\
& +\varepsilon_{1} v_{1,0}\left(\frac{\mathrm{x}-\varepsilon_{1} \mathrm{p}}{\varepsilon_{1} \varepsilon_{2}}\right)-\varepsilon_{1} v_{1,0}\left(\frac{\varsigma(\mathrm{x})-\varepsilon_{1} \mathrm{p}}{\varepsilon_{1} \varepsilon_{2}}\right) \\
& +\varepsilon_{2} v_{0,1}\left(\frac{\mathrm{x}-\varepsilon_{1} \mathrm{p}}{\varepsilon_{1} \varepsilon_{2}}\right)-\varepsilon_{2} v_{0,1}\left(\frac{\varsigma(\mathrm{x})-\varepsilon_{1} \mathrm{p}}{\varepsilon_{1} \varepsilon_{2}}\right) \\
& +\varepsilon_{1}^{2} v_{2,0}\left(\frac{\mathrm{x}-\varepsilon_{1} \mathrm{p}}{\varepsilon_{1} \varepsilon_{2}}\right)-\varepsilon_{1}^{2} v_{2,0}\left(\frac{\varsigma(\mathrm{x})-\varepsilon_{1} \mathrm{p}}{\varepsilon_{1} \varepsilon_{2}}\right) \\
& +\varepsilon_{1} \varepsilon_{2} v_{1,1}\left(\frac{\mathrm{x}-\varepsilon_{1} \mathrm{p}}{\varepsilon_{1} \varepsilon_{2}}\right)-\varepsilon_{1} \varepsilon_{2} v_{1,1}\left(\frac{\varsigma(\mathrm{x})-\varepsilon_{1} \mathrm{p}}{\varepsilon_{1} \varepsilon_{2}}\right) \\
& +\varepsilon_{2}^{2} v_{0,2}\left(\frac{\mathrm{x}-\varepsilon_{1} \mathrm{p}}{\varepsilon_{1} \varepsilon_{2}}\right)-\varepsilon_{2}^{2} v_{0,2}\left(\frac{\varsigma(\mathrm{x})-\varepsilon_{1} \mathrm{p}}{\varepsilon_{1} \varepsilon_{2}}\right) \quad \forall \mathrm{x} \in \overline{\Omega_{\varepsilon}} .
\end{aligned}
$$

\section{Fourth (and last) iteration}

Step 1. With the approximation (4.25) the main error left on $\partial_{+} \Omega$ is still given by (4.23), the misfit produced on $\partial_{+} \Omega$ by the corrector added in the third round is indeed in $O\left(\sum_{i+j=4} \varepsilon_{1}^{i} \varepsilon_{2}^{j}\right)$ and it is smaller than (4.23). The main error on $\partial \omega_{\varepsilon}$ can be computed as in the previous iterations and it is

$$
\begin{aligned}
& \varepsilon_{1}^{3}\left[u_{0,0}\right]_{3,0}^{\omega}(\mathbf{X})+\varepsilon_{1}^{2} \varepsilon_{2}\left[u_{0,0}\right]_{2,1}^{\omega}(\mathbf{X})+\varepsilon_{1}^{2} \varepsilon_{2}\left[u_{1,1}\right]_{1,0}^{\omega}(\mathbf{X})-\varepsilon_{2}^{3}\left[v_{0,0}\right]_{3}^{\omega, \varsigma(\omega)}(\mathbf{X})-\varepsilon_{1} \varepsilon_{2}^{2}\left[v_{1,0}\right]_{2}^{\omega, \varsigma(\omega)}(\mathbf{X}) \\
& \quad-\varepsilon_{2}^{3}\left[v_{0,1}\right]_{2}^{\omega, \varsigma(\omega)}(\mathbf{X})-\varepsilon_{1}^{2} \varepsilon_{2}\left[v_{2,0}\right]_{1}^{\omega, \varsigma(\omega)}(\mathbf{X})-\varepsilon_{1} \varepsilon_{2}^{2}\left[v_{1,1}\right]_{1}^{\omega, \varsigma(\omega)}(\mathbf{X})-\varepsilon_{2}^{3}\left[v_{0,2}\right]_{1}^{\omega, \varsigma(\omega)}(\mathbf{X}) \quad \forall \mathbf{X} \in \partial \omega_{\varepsilon} .
\end{aligned}
$$

Both the error on $\partial_{+} \Omega$ and the one on $\partial \omega_{\varepsilon}$ are in $O\left(\sum_{i+j=3} \varepsilon_{1}^{i} \varepsilon_{2}^{j}\right)$ and we are in case (a).

Step 2.(a). We can decide to stop here the iterative procedure. We have obtained an approximation with a remainder in $O\left(\sum_{i+j=3} \varepsilon_{1}^{i} \varepsilon_{2}^{j}\right)$ and accordingly we can write and expression for all the coefficients $U_{i, j}$ and $V_{i, j}$ with $i+j \leq 2$ (cf. Proposition 3.2). In particular, it holds $U_{1,0}=U_{0,1}=U_{2,0}=U_{0,2}=0$ and for all others we have $U_{i, j}=u_{i, j}$ and $V_{i, j}=v_{i, j}$. Moreover, since computing the extension operators $E_{\Omega}$ on a function $\phi \in \mathscr{C}^{1, \alpha}(\partial \Omega)$ and $E_{\omega}$ on a function $\psi \in \mathscr{C}^{1, \alpha}(\partial \omega)$ is equivalent to the solution of problems (4.13) and (4.14), respectively, the computation of such coefficients $U_{i, j}$ and $V_{i, j}$ is reduced to the solution of the boundary value problems corresponding to 4.18, 4.21, and 4.24.

\section{Acknowledgement}

The authors are partially supported by the ANR (Agence Nationale de la Recherche), projects ARAMIS $n^{\circ}$ ANR-12-BS01-0021. M. Dalla Riva also acknowledges the support of HORIZON 2020 MSC EF project FAANon (grant agreement MSCA-IF-2014-EF - 654795) at the University of Aberystwyth, UK. P. Musolino has received funding from the European Union's Horizon 2020 research and innovation programme under the Marie Skłodowska-Curie grant agreement No 663830. P. Musolino is a member of the Gruppo Nazionale per l'Analisi Matematica, la 
Probabilità e le loro Applicazioni of the Istituto Nazionale di Alta Matematica (INdAM). Part of the work has been carried out while P. Musolino was visiting the 'Département de mathématiques et applications' of the 'École normale supérieure, Paris'. P. Musolino wishes to thank the 'Département de mathématiques et applications' and in particular V. BonnaillieNoël for the kind hospitality.

\section{References}

[1] V. Bonnaillie-Noël, M. Dalla Riva, M. Dambrine, P. Musolino. A Dirichlet problem for the Laplace operator in a domain with a small hole close to the boundary. J. Math. Pures Appl. (9) 116 (2018) 211-267.

[2] V. Bonnaillie-Noël, M. Dambrine, S. Tordeux, G. Vial. Interactions between moderately close inclusions for the Laplace equation. Math. Models Methods Appl. Sci. 19(10) (2009) 18531882.

[3] M. Costabel, M. Dalla Riva, M. Dauge, P. Musolino. Converging expansions for selfsimilar perforations of a plane sector. Integral Equations Operator Theory 88(3) (2017) 401-449.

[4] M. Dalla Riva, P. Musolino. A mixed problem for the Laplace operator in a domain with moderately close holes. Comm. Partial Differential Equations 41(5) (2016) 812-837.

[5] M. Dalla Riva, P. Musolino, R. Pukhtaievych. Series expansion for the effective conductivity of a periodic dilute composite with thermal resistance at the two-phase interface. Asymptot. Anal. (to appear).

[6] M. Dalla Riva, P. Musolino, S. V. Rogosin. Series expansions for the solution of the Dirichlet problem in a planar domain with a small hole. Asymptot. Anal. 92(3-4) (2015) 339-361.

[7] G. B. Folland. Introduction to partial differential equations. Princeton University Press, Princeton, NJ, second edition 1995.

[8] D. Gilbarg, N. S. Trudinger. Elliptic partial differential equations of second order, volume 224 of Grundlehren der Mathematischen Wissenschaften [Fundamental Principles of Mathematical Sciences]. Springer-Verlag, Berlin, second edition 1983.

[9] A. M. IL'In. A boundary value problem for an elliptic equation of second order in a domain with a narrow slit. I. The two-dimensional case. Mat. Sb. (N.S.) 99(141)(4) (1976) 514-537.

[10] A. M. IL'IN. Matching of asymptotic expansions of solutions of boundary value problems, volume 102 of Translations of Mathematical Monographs. American Mathematical Society, Providence, RI 1992. Translated from the Russian by V. Minachin [V. V. Minakhin].

[11] M. Lanza de Cristoforis. Asymptotic behaviour of the conformal representation of a Jordan domain with a small hole in Schauder spaces. Comput. Methods Funct. Theory 2(1) (2002) 1-27.

[12] M. Lanza de Cristoforis. Asymptotic behavior of the solutions of the Dirichlet problem for the Laplace operator in a domain with a small hole. A functional analytic approach. Analysis (Munich) 28(1) (2008) 63-93.

[13] V. Maz'Ya, A. Movchan, M. Nieves. Green's kernels and meso-scale approximations in perforated domains, volume 2077 of Lecture Notes in Mathematics. Springer, Heidelberg 2013.

[14] V. Maz'ya, S. Nazarov, B. PlamenevskiJ. Asymptotic theory of elliptic boundary value problems in singularly perturbed domains. Vol. I, volume 111 of Operator Theory: Advances and Applications. Birkhäuser Verlag, Basel 2000. Translated from the German by Georg Heinig and Christian Posthoff. 
[15] T. VAlEnt. Boundary value problems of finite elasticity, volume 31 of Springer Tracts in Natural Philosophy. Springer-Verlag, New York 1988. Local theorems on existence, uniqueness, and analytic dependence on data. 